\title{
Drawer algorithms for 1-space bounded multidimensional hyperbox packing
}

\author{
Paulina Grzegorek ${ }^{1}$ (D) Janusz Januszewski ${ }^{1}$
}

Published online: 17 August 2018

(c) The Author(s) 2018

\begin{abstract}
We study a multidimensional hyperbox packing with one active bin. The items $(d-$ dimensional hyperboxes of edge length not greater than 1) arrive one by one. Each item must be packed online into a hypercube bin of edge 1 and $90^{\circ}$-rotations are allowed. If it is impossible to pack an item into an active bin, we close the bin and open a new active bin to pack that item. In this paper, we present a $3.5^{d}$-competitive as well as a $12 \cdot 3^{d}$-competitive online $d$-dimensional hyperbox packing algorithm with one active bin.
\end{abstract}

Keywords Online algorithms · Bin packing $\cdot$ Multidimensional $\cdot$ One-space bounded

Mathematics Subject Classification 68W27

\section{Introduction}

A finite sequence $S$ of items is given. When all the items of $S$ are accessible, the packing method is called offline. When items arrive one by one and each item that has arrived must be packed into a bin and cannot be moved thereafter, the packing method is called online. In the online version of packing a crucial parameter is the number of bins available for packing, i.e., active bins. It is natural to expect a packing method to be less efficient with fewer number of active bins. Online packing methods are further divided into two classes: unbounded space when no restriction on the number of active bins occurs and $t$-space bounded with the maximum of $t$ active bins at the same time.

Paulina Grzegorek

Paulina.Grzegorek@utp.edu.pl

Janusz Januszewski

januszew@utp.edu.pl

1 Institute of Mathematics and Physics, UTP University of Science and Technology,

A1. Prof. S. Kaliskiego 7, 85-789 Bydgoszcz, Poland 
In this paper we consider an online version of packing with only one active bin, i.e., a 1-space bounded model. Each item of the sequence $S$ is packed into the active bin. When the packing is not possible, the bin is closed and is never used again. A new active bin is opened.

Let $A(S)$ be the number of bins used by the algorithm $A$ to pack a sequence $S$. Let $O P T(S)$ be the number of bins used to pack items from $S$ with the most efficient offline method, i.e., the minimum number of bins containing whole sequence $S$. The asymptotic competitive ratio for algorithm $A$ is defined as:

$$
R_{A}^{\infty}=\lim _{n \rightarrow \infty} \sup _{S}\left\{\frac{A(S)}{O P T(S)} \mid O P T(S)=n\right\} .
$$

The online bin packing is a classical problem studied for more than 40 years. Onedimensional bin packing was first investigated in Ullman (1971) (see also Johnson et al. 1974), where the performance ratio of the First Fit algorithm was proved to be 17/10. The analysis of the Next Fit algorithm can be found in Johnson (1974), where the author shows that the performance ratio is not greater than 2. Revised First Fit presented in Yao (1980) has performance ratio 5/3. The article also gives the lower bound $3 / 2$ of one dimensional online bin packing. The improvement of this result can be found in Brown (1979) and Liang (1980), who proved that the lower bound is not smaller than 1.53635. Currently the best know lower bound is 1.54014 , proved by van Vliet (1992).

Concerning the two-dimensional online bin packing algorithms Coppersmith and Raghavan (1989) presented the algorithm with competitive ratio 3.25. The result was later improved by Csirik et al. (1993) to 3.0625 and by Han et al. (2001) to 2.7834 . Further improvements can be found in Seiden and van Stee (2003), where the authors show the upper bound 2.66013 of the asymptotic competitive ratio. The upper bound currently stands at 2.5545 (see Han et al. 2011).

One can also consider general bounded space packing methods, where the number of active bins is finite, but not specified. Lee and Lee (1985) presented the Harmonic algorithm with competitive ratio not greater than 1.63597. Ramanan et al. (1989) showed that the upper bound can be improved to 1.61217 and gave the lower bound 1.58333. Seiden (2002) further improved the upper bound to 1.58889. The best know upper bound 1.5813 is proved in Heydrich and van Stee (2016).

In optimal algorithms (Harmonic algorithm and its improvements) when the asymptotic competitive ratio approaches the optimal value, the number of active bins diverges to infinity. It is hard to expect to use this result in practical applications. Thus a question arises: What asymptotic competitive ratio can we achieve when the number of active bins is bounded above by a given (small) natural number? This question was addressed by Woeginger (1993) whose Simplified Harmonic 6-space bounded online algorithm has competitive ratio beneath $17 / 10$.

Different types of items in the sequence $S$ can be considered. In $d$-dimensional bin packing problem a bin is a unit hypercube and each item of $S$ is a hyperbox of edge lengths not greater than 1 . Items can be rotated by $90^{\circ}$ in any plane defined by arbitrary two of the item's edges. 
For $d$-dimensional hyperbox packing Epstein and van Stee $(2005)$ gave a $\left(\Pi_{\infty}\right)^{d}$ competitive space bounded algorithm, where $\Pi_{\infty} \approx 1.69103 \ldots$ is the competitive ratio of the one-dimensional harmonic algorithm, see Lee and Lee (1985). Algorithms with only one active bin and 2-dimensional items were presented for the first time in Zhang et al. (2010b), where a method with competitive ratio 8.84 was given. A paper by Zhang et al. (2010a) gives an online packing algorithm with competitive ratio 5.155 for rectangles and a 4.5-competitive algorithm for squares. Another result was obtained by Zhang et al. (2014) with competitive ratios 5.06 and 4.3 achieved for squares. Also a paper by Grzegorek and Januszewski (2015) presents a 3.883-competitive online square packing algorithm. In an article by Januszewski and Zielonka (2018) the authors describe a 4.84-competitive 1-space bounded 2-dimensional bin packing algorithm and present the lower bound of 3.246 for the competitive ratio. In a paper by Januszewski and Zielonka (2016) the reader can find a 3.8165-competitive 2space bounded algorithm for rectangles and a 3.6-competitive model for squares. A 3-space bounded 3.577-competitive square packing method is given in Grzegorek and Januszewski (2014). The $d$-dimensional case of one-space bounded hyperbox packing is considered in Zhang et al. (2013). The authors give an online algorithm with competitive ratio equal to $4^{d}$. Two-space bounded hypercube packing with competitive ratio $32 / 21 \cdot 2^{d}$ is discussed in Zhao and Shen (2015). Online packing of $d$-dimensional hypercubes with total volume not greater than $(n+1) 2^{-d}$ into $n$ unit $d$-dimensional hypercubes is considered in Zielonka (2016).

We focus on the problem of online packing of $d$-dimensional hyperboxes into one active bin. The paper contains two algorithms $D_{1}(d)$ and $D_{2}(d)$ : the first method is a $3.5^{d}$-competitive algorithm and for $d<17$ works better than the second algorithm having the $12 \cdot 3^{d}$ competitive ratio, which is a significant improvement of the ratio $4^{d}$ from Zhang et al. (2013). Both algorithms are defined inductively from lower dimensions to higher. The inductive step goes two dimensions back and thus the core of the algorithm is the method of packing rectangles on the front wall of the unit hypercube. Since in three-dimensions this looks a lot like drawers we decided to name it: the drawer method. As a base, for $d=1$ both algorithms take the Next Fit algorithm and for $d=2$ the 1-space bounded algorithm from Zhang et al. (2014).

\section{Intuitions on how algorithms work}

We give several examples of packing some 'easy' items to introduce the reader to general rules of packing used by both algorithms. Since it is difficult to handle items of completely arbitrary size, we decided to assign two-dimensional items into categories, called $\lambda$-rectangles (and their analogue $r$-rectangles for the second algorithm) depending on lengths of their edges. $\lambda$-rectangles also differ in size, however there are only countably many of them.

Each drawer is a hyperbox with $d-2$ edges of length 1 and two edges forming a $\lambda$-rectangle. Thus to define a drawer we only need to provide the size of the front wall, i.e., lengths of the two smallest edges of the hyperbox. An algorithm for choosing the right place for a drawer is described in Sect. 3: $\Lambda_{1}$-method for packing $\lambda$-rectangles. 


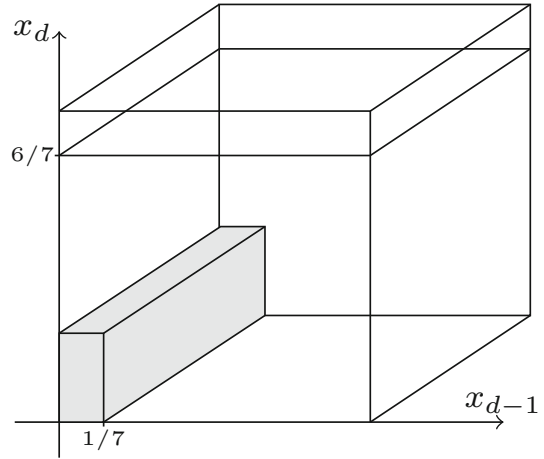

(a)

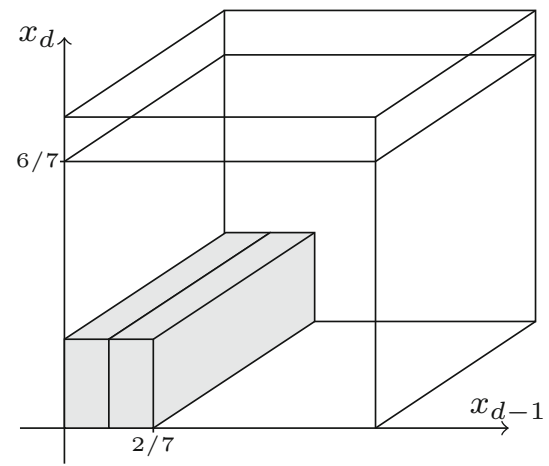

(b)

Fig. 1 The packing of the first two items. a A bin with the first packed item. $\mathbf{b}$ A bin with two packed items
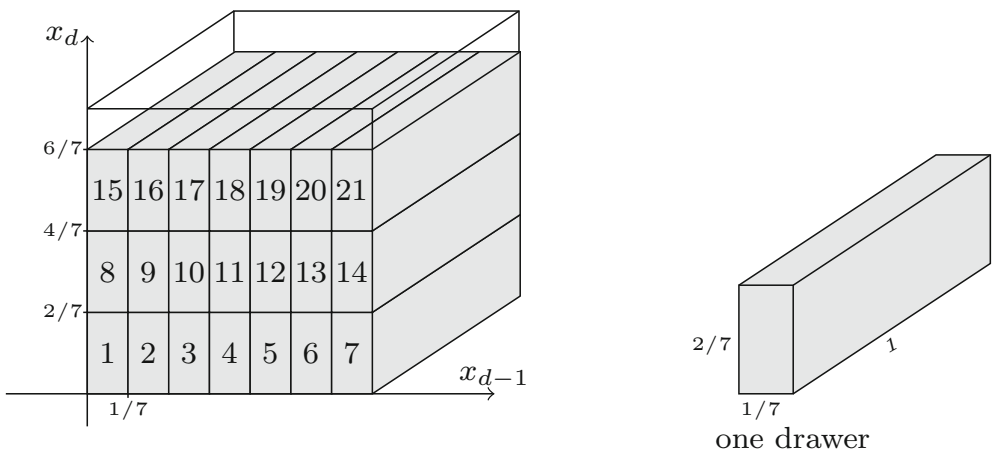

Fig. 221 hyperboxes $1^{d-2} \times 1 / 7 \times 2 / 7$

During packing, there are no empty drawers in the bin (a hypercube of edge 1). A new drawer is opened only when an item arrives and there is no place to pack it in matching drawers opened earlier. Two smallest edges of the item are taken into account and a drawer with appropriate front wall is created. The item is packed into the new drawer immediately. The rest of the bin is not divided into drawers until a need for a specific drawer occurs.

In the following examples $\lambda=2 / 7$. We chose such $\lambda$ to balance the average packing ratio of big and small (see Sect. 4 for the definitions) hyperboxes for the $D_{1}(d)$-algorithm.

In Examples 1-3 each drawer is entirely packed because the items are as big as drawers. Examples 4-6 could picture an actual situation where incoming items are of arbitrary size and do not fill entire drawers.

Example 1 The packing of 21 congruent hyperboxes $1^{d-2} \times 1 / 7 \times 2 / 7$, see Figs. 1 and 2. Front walls of the hyperboxes are $\lambda$-rectangles $1 / 7 \times 2 / 7$. Upon arrival of each hyperbox a new drawer of opened. Each item is packed into an individual drawer. 

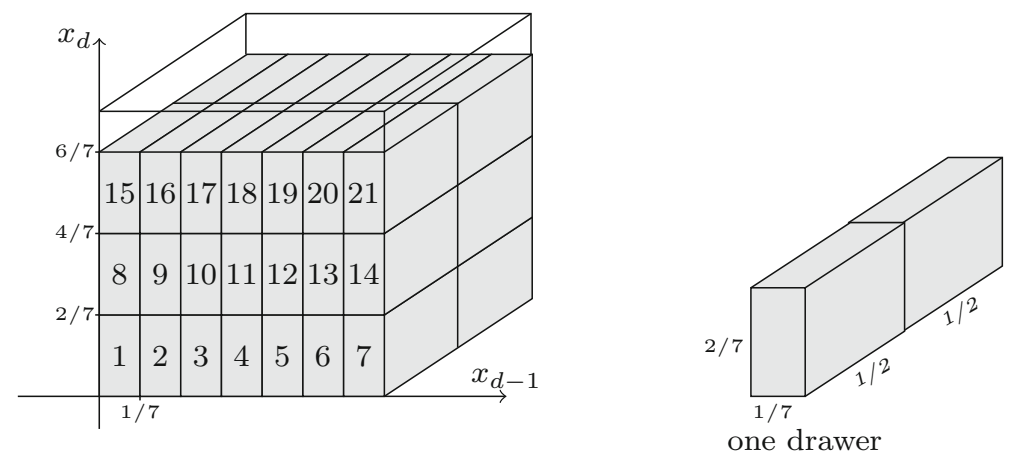

Fig. 342 hyperboxes $1^{d-3} \times 1 / 2 \times 1 / 7 \times 2 / 7$
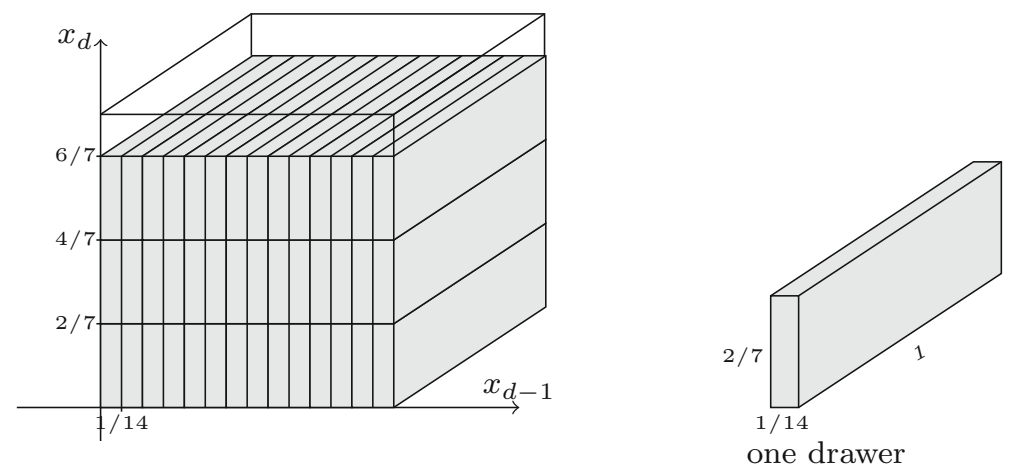

Fig. 442 hyperboxes $1^{d-2} \times 1 / 14 \times 2 / 7$

Example 2 The packing of 42 congruent hyperboxes $1^{d-3} \times 1 / 2 \times 1 / 7 \times 2 / 7$, see Fig. 3. Each drawer is packed with two items.

Example 3 The packing of 42 congruent hyperboxes $1^{d-2} \times 1 / 14 \times 2 / 7$, see Fig. 4 . Front walls of the hyperboxes are $\lambda$-rectangles. For each item an appropriate drawer is created and the item is packed into it.

The following examples are for $d=3$.

Example 4 The packing of 21 congruent boxes $0.6 \times 0.1 \times 0.2$, see Fig. 5 . In this example lengths of edges of the front wall of each box satisfy: $1 / 14<0.1<1 / 7$ and $1 / 7<0.2<2 / 7$. If we let $H=0.6 \times 0.1 \times 0.2$, then the smallest $\lambda$-rectangle $P(H)$ containing the front wall of $H$ (see Sect. 4 for precise definitions) is $1 / 7 \times 2 / 7$. For each item a new drawer with front wall $P(H)$ is opened. Each item is packed into an individual drawer.

Example 5 The packing of 42 congruent boxes $0.4 \times 0.1 \times 0.2$, see Fig. 6 . This example is a mix of Examples 2 and 4. If we let $H=0.4 \times 0.1 \times 0.2$, then the smallest $\lambda$ rectangle $P(H)$ containing the front wall of $H$ (see Sect. 4 for precise definitions) is 

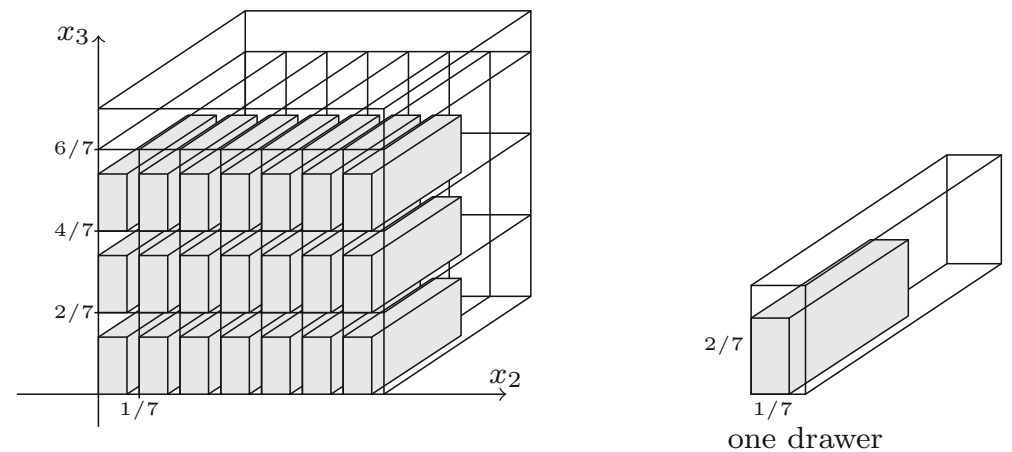

Fig. 521 boxes $0.6 \times 0.1 \times 0.2$
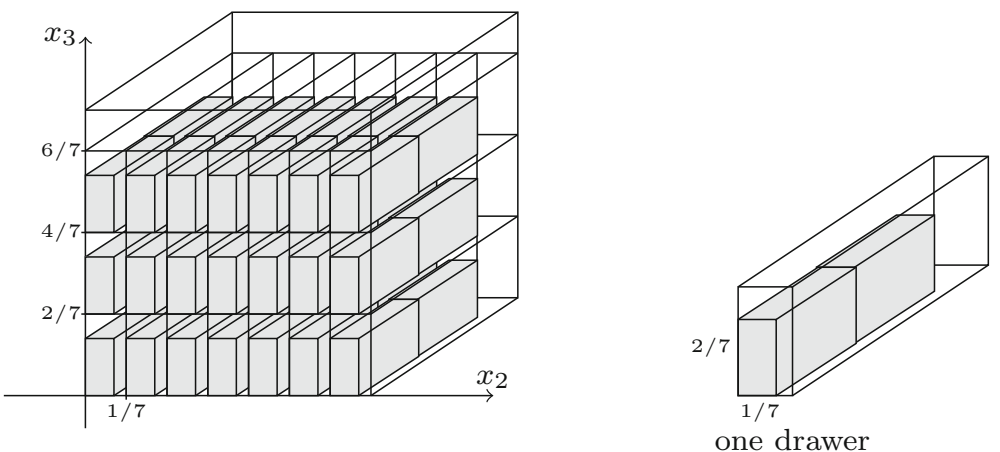

Fig. 642 boxes $0.4 \times 0.1 \times 0.2$

$1 / 7 \times 2 / 7$. Items are packed into drawers $1 \times 1 / 7 \times 2 / 7$. It is possible to fit two items in one drawer, thus each drawer is packed with two boxes $0.4 \times 0.1 \times 0.2$.

Example 6 The packing of 56 boxes: 26 boxes $0.45 \times 0.1 \times 0.25$ (we name them thick) and 30 boxes $0.35 \times 0.06 \times 0.2$ (we name them slim), see Fig. 7 . The order of arrival of items is the following: 8 thick boxes, 24 slim boxes, 14 thick boxes, 6 slim boxes and 4 thick boxes.

Two types of drawers were created. Since $1 / 7<0.25<2 / 7$ and $1 / 14<0.1<1 / 7$ each thick box is packed into a drawer with the front wall $1 / 7 \times 2 / 7$, two thick boxes per drawer. Since $1 / 7<0.2<2 / 7$ and $1 / 28<0.06<1 / 14$ each slim box is packed into a drawer with the front wall $1 / 14 \times 2 / 7$, again two slim boxes per drawer.

\section{$3 \Lambda_{1}$-method for packing $\lambda$-rectangles}

Let $\lambda>0$ and let $k$ be a non-negative integer. A $\lambda_{k}$-unit is a rectangle with height $\lambda / 2^{k}$ and width $\lambda / 2^{k+1}$. A basic unit is $\lambda_{0}$-unit. See Fig. 8 .

For basic units we consider rectangles with side ratio 1:2. It makes possible to pack a square in a union of two such rectangles. During packing basic units are divided into 

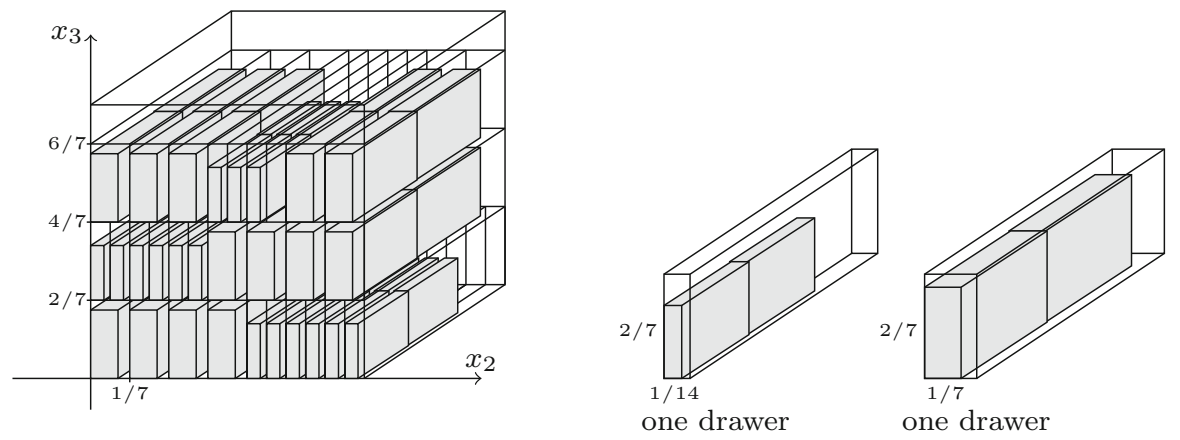

Fig. 726 boxes $0.45 \times 0.1 \times 0.25$ and 30 boxes $0.35 \times 0.06 \times 0.2$
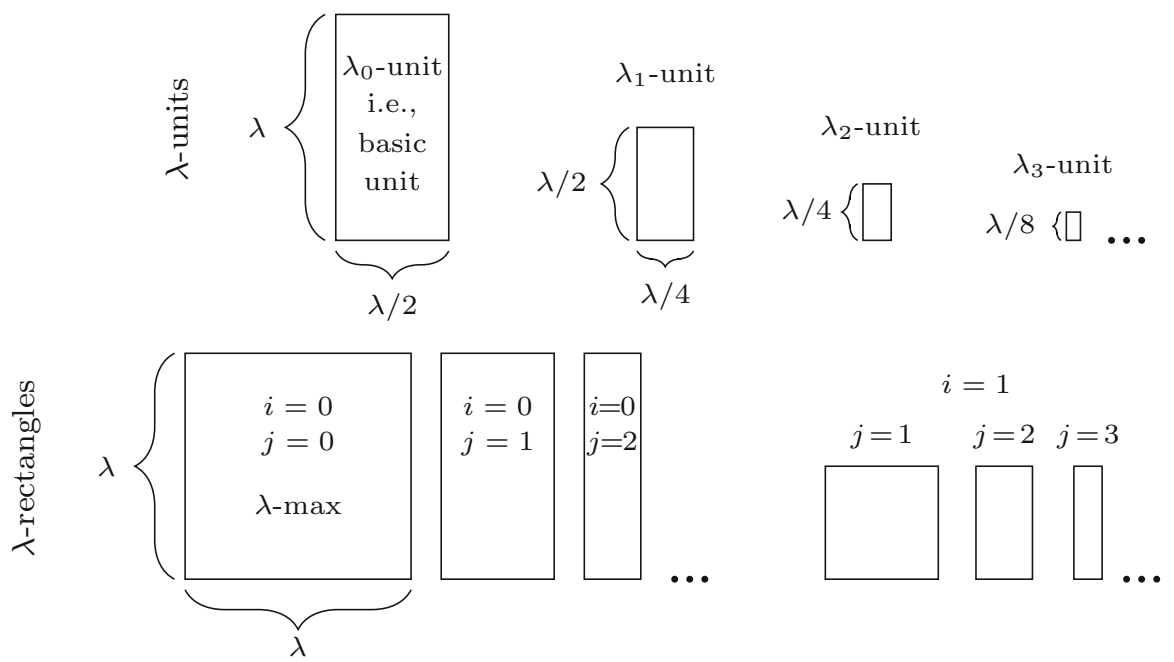

Fig. $8 \lambda$-units and $\lambda$-rectangles

smaller rectangles. Every division creates four congruent rectangles, each one being similar to a basic unit.

Consider a square $I=1 \times 1$. For $\epsilon>0$ the square $I$ is divided into two rectangles $I_{\epsilon}=1 \times(1-\epsilon)$ and $T_{\epsilon}=1 \times \epsilon$. The rectangle $T_{\epsilon}$ will be used for packing big items, while $I_{\epsilon}$-for small items (definitions can be found in Sect. 4).

Small items are packed into basic units $\lambda / 2 \times \lambda$ and to obtain high packing ratio, we wish to fit in $I_{\epsilon}$ as many basic units as possible. Of course, we also want to divide the whole $I_{\epsilon}$, therefore the widths of basic units in one row must sum up to 1 . For $D_{1}(d)$ algorithm the following values are sufficient: $\epsilon=1 / 7$ and $\lambda=2 / 7$. The rectangle $I_{\epsilon}$ (called $B_{1}$ in this case) with edges $1 \times(1-1 / 7)=3.5 \lambda \times 3 \lambda$ is divided into 21 basic units $\lambda / 2 \times \lambda$.

Suppose we pack rectangles $R_{1}, R_{2}, \ldots$ with side lengths smaller than or equal to $2 / 7$ into $B_{1}$. For each $R_{i}$ we find the smallest $\lambda$-rectangle containing $R_{i}$ (the area of 
Fig. 9 The rectangle $B_{1}$

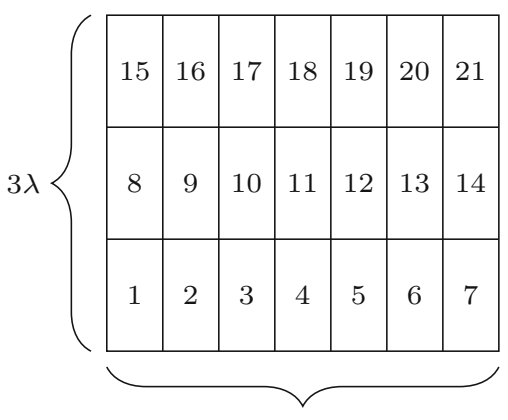

$3.5 \lambda$

Fig. 10 The division of a

$\lambda_{k-1}$-unit into four $\lambda_{k}$-units

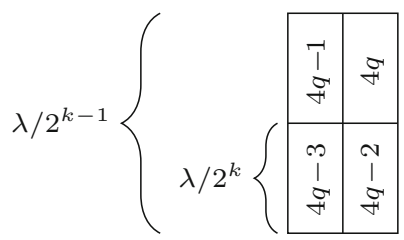

this $\lambda$-rectangle is smaller than the area of four $R_{i}$ 's). Then this $\lambda$-rectangle (along with $R_{i}$ inside) is packed into $B_{1}$.

In the next section we will pack $d$-dimensional hyperboxes into $d$-dimensional drawers. The first stage is finding the proper drawer: since we consider only front walls (of the hyperbox and of the drawer) it is exactly the same as packing rectangles with side lengths smaller or equal to $2 / 7$ into $B_{1}$. That is why the method of packing $\lambda$-rectangles into $B_{1}$ is crucial in $D_{1}(d)$-algorithm.

We decided to describe the general case with an arbitrary $\lambda$, since some of the reasoning is used again in Sect. 5 for $\lambda=1 / 3$.

Denote by $B_{1}$ a rectangle $3.5 \lambda \times 3 \lambda$ divided into twenty one basic units numbered with natural numbers in the order showed on Fig. 9. During the packing process basic units will be divided into smaller units. When a $\lambda_{k-1}$-unit (for $k \geq 1$ ) numbered with $q$ is partitioned into four $\lambda_{k}$-units, these $\lambda_{k}$-units are numbered from $4 q-3$ to $4 q$ as on Fig. 10.

Let $\lambda$-rectangle be a rectangle of width $\lambda_{j}=\lambda / 2^{j}$ and height $\lambda_{i}=\lambda / 2^{i}$ for some $0 \leq i \leq j$. $\lambda$-max is a square of side length $\lambda$ (see Fig. 8). A unit is called empty, if its interior has an empty intersection with any packed $\lambda$-rectangle.

\section{$\boldsymbol{\Lambda}_{\mathbf{1}}$-method of packing $\boldsymbol{\lambda}$-rectangles into $\mathbf{B}_{\mathbf{1}}$}

1. A $\lambda$-rectangle of height $\lambda$ and width less then $\lambda$ is packed as much to the left as possible into the lowest indexed basic unit in $B_{1}$ that has enough empty space.

2. $\lambda$-max is packed into the union of two consecutive, empty, lowest indexed basic units. Clearly, $\lambda$-max cannot be packed into units 7 and 8 or into units 14 and 15 .

3. A $\lambda$-rectangle of height $\lambda_{i}=\lambda / 2^{i}, i \geq 1$ that is not a square is packed as much to the left as possible into the lowest indexed $\lambda_{i}$-unit and, obviously, with enough empty space.

If there is no such unit, find the greatest $k \leq i$ such that there is an empty $\lambda_{k}$-unit. 


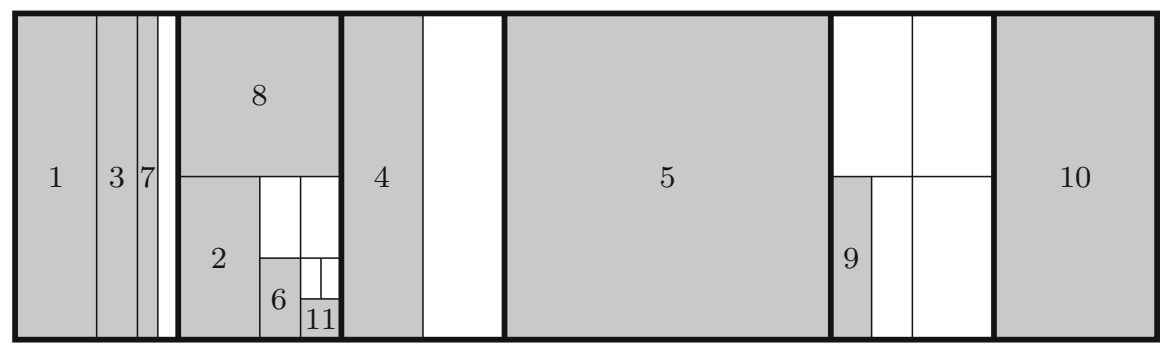

Fig. 11 Packing items into basic units $1-7$ by $\Lambda_{1}$-method. Numbers indicate the order in which the items arrive

From among empty $\lambda_{k}$-units choose the lowest indexed one and then divide it into four $\lambda_{k+1}$-units. If $\lambda_{k+1}>\lambda_{i}$, then the lowest indexed $\lambda_{k+1}$-unit is divided again into four smaller units. The division is repeated until a $\lambda_{i}$-unit is created. Now the $\lambda$-rectangle is packed into the lowest indexed $\lambda_{i}$-unit as much to the left as possible.

4. A $\lambda$-rectangle that is a square of side length $\lambda_{i}, i \geq 1$, is packed, if possible, into two empty $\lambda_{i}$-units, that were created through the division of one $\lambda_{i-1}$-unit. If there are no such units the division of a bigger unit is conducted as described in the previous case. Finally a $\lambda$-rectangle is packed into two lowest indexed $\lambda_{i}$-units (such that the union of these units is a square).

Example 7 Figure 11 illustrates $\Lambda_{1}$-method. The first $\lambda$-rectangle, by Rule 1 , is packed into the first basic unit as much to the left as possible. The second $\lambda$-rectangle is packed by Rule 3: it must be packed into a $\lambda_{1}$-unit. These units are created through the division, see Fig. 10, solely from empty units, thus we cannot take the first basic unit. The second basic unit is divided and the second item is packed into it. The third item is packed by Rule 1 and so is the fourth: we look for a basic unit with enough empty space. The fifth item is $\lambda$-max, therefore by Rule 2 it is packed into the union of two consecutive, empty, lowest indexed basic units. To pack the sixth item we use Rule 3: a division of an empty, lowest indexed (which would be 6 in this example) $\lambda_{1}$-unit is conducted and the item is packed. The seventh item can be fitted into the first basic unit (Rule 1). The eighth $\lambda$-rectangle is packed by Rule 4 into two empty $\lambda_{1}$-units contained in the second basic unit. Since after this packing there is no empty $\lambda_{1}$-unit left to pack the ninth item, we perform a new division (Rule 3). Enough empty space for the tenth item is only in a new, empty basic unit. The last, eleventh item is packed by Rule 4 into freshly created $\lambda_{3}$-units from the lowest indexed $\lambda_{2}$-unit.

$\lambda$-rectangles shown on Fig. 11 can be front walls of drawers with $(d-2)$ edges of length 1 (see Sect. 4).

Lemma 1 Let $k \geq 3$ and let a $\lambda$-rectangle of width smaller then $\lambda$ be packed into the unit number $k$ in $B_{1}$. The empty space in units numbered from 1 to $k$ is smaller than $7 \lambda^{2} / 6$.

Proof First, we will show that the empty space in all units that are partially packed is smaller than $\frac{2}{3} \lambda^{2}$. 

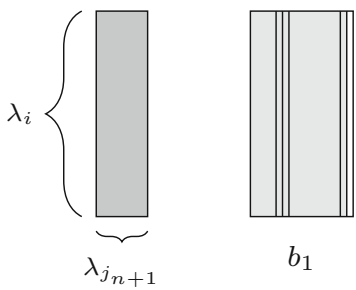

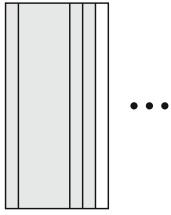

$b_{2}$

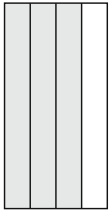

$b_{n}$

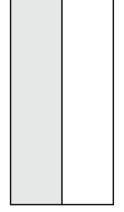

$b_{n+1}$

Fig. 12 Units of height $\lambda_{i}$

Let $i \geq 0$. Consider all units of height $\lambda_{i}$ (and width $\frac{1}{2} \lambda_{i}$ ) into which an item of height $\lambda_{i}$ was packed. Let $b_{n+1}$ be the last such unit. From among remaining units (different from $b_{n+1}$ ) we choose units $b_{1}, b_{2}, \ldots, b_{n}$ that are not entirely packed (see Fig. 12). Let $R_{j_{i}}$ be an item with the smallest width $\lambda_{j_{i}}$ (and of height $\lambda_{i}$ ) packed into $b_{i}$ for $i=1, \ldots, n+1$. The width of empty space in $b_{n}$ is smaller than $\lambda_{j_{n+1}}$, otherwise $R_{j_{n+1}}$ can be packed into this unit. This implies that $\lambda_{j_{n}}<\lambda_{j_{n+1}}$ (a rectangle of width smaller than $\lambda_{j_{n+1}}$ was packed into $b_{n}$ ). Since the width of empty space in $b_{n}$ must be a multiple of $\lambda_{j_{n}}$ (the width of $b_{n}$ as well as the width of each item packed into $b_{n}$ is a multiple of $\lambda_{j_{n}}$ ), it follows that the width of empty space in $b_{n}$ is not greater than $\lambda_{j_{n+1}}-\lambda_{j_{n}}$. For the same reason the width of empty space in $b_{n-1}$ is not greater than $\lambda_{j_{n}}-\lambda_{j_{n-1}}$. Repeating this argument, we get that the sum of widths of empty space in all $b_{1}, b_{2}, \ldots, b_{n}$ is not greater than

$$
\lambda_{j_{n+1}}-\lambda_{j_{n}}+\lambda_{j_{n}}-\lambda_{j_{n-1}}+\cdots+\lambda_{j_{2}}-\lambda_{j_{1}}<\lambda_{j_{n+1}} .
$$

The empty space in $b_{n+1}$ is not greater than the area of this unit $\left(\lambda_{i}^{2} / 2\right)$ minus the area of $R_{j_{n+1}}$. Consequently, the empty space in all units $b_{1}, \ldots, b_{n+1}$ (of height $\lambda_{i}$ ) is less than

$$
\lambda_{j_{n+1}} \cdot \lambda_{i}+\frac{1}{2} \lambda_{i}^{2}-\lambda_{j_{n+1}} \cdot \lambda_{i}=\frac{1}{2} \lambda_{i}^{2}
$$

Finally, the empty space in partially packed units of all heights is not greater than

$$
\sum_{i \geq 0} \frac{1}{2} \lambda_{i}^{2}=\frac{1}{2} \cdot \lambda^{2} \sum_{i \geq 0} \frac{1}{4^{i}}=\frac{1}{2} \lambda^{2} \cdot \frac{4}{3}=\frac{2}{3} \lambda^{2} .
$$

Entirely packed units do not add anything to the empty space, therefore they are omitted.

Now, we will calculate the number of empty units of all sizes. There is no empty basic unit, otherwise it would be used for packing. Smaller units are created during the division to pack smaller items. At each step four units are created, but at least one unit is immediately used either for the next division or for packing. Thus at most 3 units of height smaller than $\lambda$ can be empty. This gives 


$$
3 \cdot \sum_{i \geq 1} \frac{1}{2} \lambda_{i}^{2}=\frac{3}{2} \lambda^{2} \cdot \sum_{i \geq 1} \frac{1}{4^{i}}=\frac{1}{2} \lambda^{2} .
$$

The empty space in all basic units numbered from 1 to $k$ is less than

$$
\frac{2}{3} \lambda^{2}+\frac{1}{2} \lambda^{2}=\frac{7}{6} \lambda^{2}
$$

Lemma 2 Let $R$ be $\lambda$-max. If $R$ is packed into $B_{1}$ into the union of two consecutive units numbered $k-1$ and $k$, where $2 \leq k \leq 21$ and, obviously, $k \neq 8$ as well as $k \neq 15$, then the empty space in units 1 to $k$ is not greater than

$-13 \lambda^{2} / 6$, for $k \in\{16,18,19,20,21\}$

$-5 \lambda^{2} / 3$, for $k \in\{9,11,13\}$,

$-7 \lambda^{2} / 6$, for $k \in\{2,3,4,5,6,7,10,12,14,17\}$.

Moreover, if there is not enough space to pack $\lambda$-max into $B_{1}$, then the empty space in $B_{1}$ is smaller than $8 \lambda^{2} / 3$.

Proof Bottom row, $2 \leq k \leq 7$

If $R$ is packed in the bottom row, there are no empty basic units preceding $R$, thus by Lemma 1 we get that the empty space is not greater than $7 \lambda^{2} / 6$.

Middle row, $9 \leq k \leq 14$

Case M1 $R$ is packed into units $8-9$. Use Lemma 1 or the case above to show that when unit 7 is

- occupied by a $\lambda$-rectangle, then the empty space is at most $7 \lambda^{2} / 6$,

- empty, then unit 6 is not empty (otherwise $R$ can be packed into the union of two consecutive units 6 and 7). Empty space is at most

$$
\frac{7}{6} \lambda^{2}+\frac{1}{2} \lambda^{2}=\frac{5}{3} \lambda^{2}
$$

Case M2 $R$ is packed into units $9-10$. Unit 8 contains a smaller $\lambda$-rectangle, thus by Lemma 1 the empty space in units 1 to 10 is not greater than $7 \lambda^{2} / 6$.

Case M3 $R$ is packed into units 10-11 or 12-13. If $R$ is packed into units $12-13$ and unit 11 does not contain $\lambda$-max, then the empty space is smaller then $7 \lambda^{2} / 6$. If units 10-11 contain $\lambda$-max , then consider the contents of unit 9 . If there is a smaller $\lambda$-rectangle use Lemma 1 ( $7 \lambda^{2} / 6$ of the empty space), otherwise use Case M1. The empty space does not exceed $5 \lambda^{2} / 3$.

Case M4 $R$ is packed into units 11-12 or 13-14. Use the similar reasoning as in the Case M3. By Lemma 1 and Case M2 the empty space does not exceed $7 \lambda^{2} / 6$.

Upper row, $k \geq 16$

Case U1 ג-max is packed into units 15-16. 

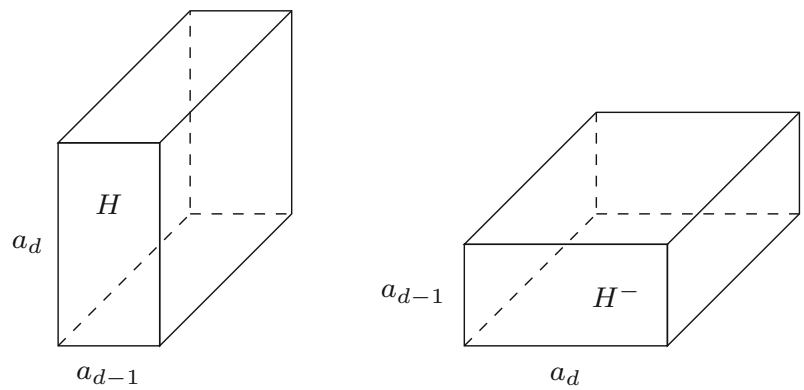

Fig. $13 H$ and $H^{-}$

- If unit 14 contains a $\lambda$-rectangle, then by Lemma 1 or Case M4 there is at most $7 \lambda^{2} / 6$ empty space.

- If unit 14 is empty, then unit 13 is not empty. It contains either $\lambda$-max (Case M3) or a smaller $\lambda$-rectangle. Consequently, the empty space is at most $5 \lambda^{2} / 3+\lambda^{2} / 2=$ $13 \lambda^{2} / 6$.

Case U2 $\lambda$-max is packed into units 16-17. Unit 15 must be packed with a smaller $\lambda$-rectangle. By Lemma 1 the empty space is less than $7 \lambda^{2} / 6$.

Case U3 $\lambda$-max is packed into two consecutive units from 17-21. Use Lemma 1 or Case U1 or Case U2. The empty space is less than $13 \lambda^{2} / 6$.

Case E: There is not enough space to pack $\lambda$-max into $B_{1}$. The worst case is when unit 21 is empty. Empty space in $B_{1}$ does not exceed $13 \lambda^{2} / 6+\lambda^{2} / 2=8 \lambda^{2} / 3$.

\section{First drawer algorithm}

Let $\lambda=2 / 7$. In this section a drawer algorithm $D_{1}(d)$ with the asymptotic competitive ratio not greater than $3.5^{d}$ is presented.

The value of $\lambda$ was chosen to ensure that the average packing ratios of big and small items were similar to each other and were both not smaller than $(2 / 7)^{d}$.

The ratio of packing big items is of the form $c \cdot \lambda^{d}$, thus smaller $\lambda$ gives smaller packing ratio. On the other hand, if $\lambda$ is greater than $2 / 7$, then $I_{\epsilon}$ contains less basic units. Moreover the volume of open drawers (in the proof of Theorem 1 it is showed to be bounded from above by $8 \lambda^{2} / 3$ ) and the empty space (see Lemmas 1 and 2) increases. The average occupation in closed drawers would be smaller and consequently the packing ratio of small items is less than $(2 / 7)^{d}$.

Each hyperbox $H$ is rotated to satisfy

$$
a_{1} \geq \cdots \geq a_{d-2} \geq a_{d} \geq a_{d-1}
$$

where $a_{j}$ is the length of the $j$ th edge of $H$ (see Fig. 13, left).

If $W$ is a hyperbox $\left[v_{1}, w_{1}\right] \times \cdots \times\left[v_{d}, w_{d}\right]$, i.e., $W=\left\{\left(x_{1}, \ldots, x_{d}\right): v_{1} \leq x_{1} \leq\right.$ $\left.w_{1}, \ldots, v_{d} \leq x_{d} \leq w_{d}\right\}$, then by the front wall of $W$ we mean the set of its points 


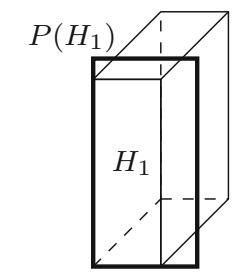

$H_{1}$ is of type $(0,1)$

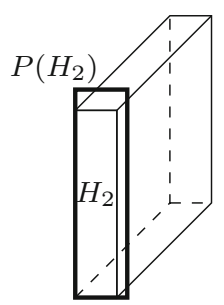

$H_{2}$ is of type $(0,2)$

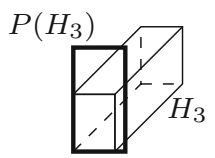

$H_{3}$ is of type $(1,2)$

Fig. 14 Hyperboxes $H_{i}$ and their $\lambda$-rectangles $P\left(H_{i}\right)$

with $x_{1}=v_{1}, \ldots, x_{d-2}=v_{d-2}$. Without loss of generality we can assume that the active bin is $[0,1]^{d}$. Let $B_{1}(d)=[0,1]^{d-1} \times[0,6 / 7]$. Obviously, the front wall of $B_{1}(d)$ is the set of its points with $x_{1}=\cdots=x_{d-2}=0$.

A hyperbox with $a_{d}>\lambda$ is called big, otherwise it is called small. Note that big hyperboxes fulfill $a_{1} \geq \cdots \geq a_{d-2} \geq a_{d}>\lambda$, while small hyperboxes satisfy $a_{d-1} \leq a_{d} \leq \lambda$.

For each small hyperbox $H$, let $P(H)$ be the smallest $\lambda$-rectangle containing the front wall of $H$. There are integers $i$ and $j$ such that the height of $P(H)$ equals $\lambda / 2^{i}$, the width of $P(H)$ equals $\lambda / 2^{j}$ and moreover $\lambda / 2^{i+1}<a_{d} \leq \lambda / 2^{i}$ and $\lambda / 2^{j+1}<a_{d-1} \leq \lambda / 2^{j}$, see Fig. 14. We say then that $H$ is of type $(i, j)$.

Using the division of $B_{1}$, the hyperbox $B_{1}(d)$ can be divided into 21 hyperboxes $1 \times \cdots \times 1 \times 1 / 7 \times 2 / 7$ that will be called basic drawers or drawers (see Fig. 17). During the packing process each drawer can be divided into smaller drawers, i.e., hyperboxes of edges $1 \times \cdots \times 1 \times \lambda / 2^{j} \times \lambda / 2^{i}$ called $(i, j)$-drawers. As described above, the front wall of any $(i, j)$-drawer is a rectangle $\lambda / 2^{j} \times \lambda / 2^{i}$ and $B_{1}$ is the front wall of $B_{1}(d)$.

Drawers are created for packing items: there can be drawers of different sizes and many drawers of the same size, however at most one drawer of a fixed size is open at each stage of the packing process. Moreover, any two (open or closed) drawers have disjoint interiors. For example, if we treat the numbered rectangles on Fig. 11 as the front walls of all drawers used for the packing, then drawers with numbers $2, \ldots, 10$ are open while the drawer with number 1 must be closed (its size is equal to the size of the drawer with number 4). Let us add that the proper drawer for a hyperbox with $1 / 7<a_{d-1} \leq a_{d} \leq 2 / 7$ is the union of two adjacent basic drawers (see 5 on Fig. 11).

Since the $D_{1}(d)$-algorithm is defined inductively two dimensions back, for $d=1$ and $d=2$ we use some already known algorithms. The exact method of packing is not crucial, we only need to achieve a certain average occupation. For $d=1$, we chose the Next Fit algorithm as the $D_{1}(1)$-algorithm, i.e., we pack any item into the active bin as much to the left as it is possible. Clearly, the average occupation in each bin is greater than $1 / 2$, however any other method with the average occupation greater than $1 / 3.5$ is also suitable. For $d=2$, as $D_{1}(2)$-algorithm we use the method described in Zhang et al. (2014) (average occupation greater than 0.197). Again, the reader can take any other 1-space bounded packing algorithm with the average occupation greater than $1 /(3.5)^{2} \approx 0.0816$ 
Assume that $d \geq 3$.

If $H=a_{1} \times \cdots \times a_{d-2} \times a_{d-1} \times a_{d}$ is a hyperbox such that $a_{1} \geq \cdots \geq a_{d-2} \geq$ $a_{d} \geq a_{d-1}$, then $H^{-}=a_{1} \times \cdots \times a_{d-2} \times a_{d} \times a_{d-1}$ is the image of $H$ in rotation of $90^{\circ}$ on the plane $x_{d-1} x_{d}$ (see Fig. 13). By the height $h$ of $H^{-}$we mean $a_{d-1}$. Clearly, $h=\min \left(a_{1}, \ldots, a_{d}\right)$ and $h$ is the length of the edge of $H^{-}$parallel to the $x_{d}$-axis.

If $W$ is a hyperbox $\left[v_{1}, w_{1}\right] \times \cdots \times\left[v_{d}, w_{d}\right]$, then by the the top of $W$ we mean the set of its points with $x_{d}=w_{d}$. The $(d-2)$-dimensional bottom of $W$ is the set of its points with $x_{d}=v_{d}$ and $x_{d-1}=v_{d-1}$. We say that $W$ is packed along the right edge of the bin $[0,1]^{d}$ provided $W$ is contained in the bin and there is $p_{d} \in$ $\left[0,1-w_{d}+v_{d}\right]$ such that $W$ contains the segment with endpoints $\left(0, \ldots, 0,1, p_{d}\right)$ and $\left(0, \ldots, 0,1, p_{d}+w_{d}-v_{d}\right)$.

\section{$\mathbf{D}_{1}(\mathrm{~d})$-algorithm of packing a hyperbox $\mathrm{H}$}

- If $H$ is big, it is rotated to obtain $H^{-}$. Then $H^{-}$is packed into the active bin from top to bottom along the right edge of the bin (see Fig. 17) provided the interior of the packed hyperbox is disjoint with those basic drawers that already contain small hypercubes. If that is not possible, we close the active bin and open a new active bin to pack $H$.

- If $H$ is a small hyperbox of type $(i, j)$, then

- it is packed into the open $(i, j)$-drawer in such a way that the $(d-2)$ dimensional bottom of $H$ is packed into the $(d-2)$-dimensional bottom of the drawer (i.e., is packed into the $(d-2)$-dimensional unit hypercube) using the $D_{1}(d-2)$-algorithm.

- If there is not enough empty space in the open $(i, j)$-drawer to pack $H$, close this drawer. A new drawer is opened in the following way. First, determine the proper place $Q \subset B_{1}$ to pack the rectangle $P(H)$ into $B_{1}$ by $\Lambda_{1}$-method.

- If the drawer with the front wall equal to $Q$ is disjoint with the interior of any packed big hypercube, then the new open $(i, j)$-drawer is the one with the front wall equal to $Q$. The rectangle $Q \subset B_{1}$ is treated as a $\lambda$-rectangle packed into $B_{1}$ in $\Lambda_{1}$-method. The hyperbox $H$ is packed into this open $(i, j)$-drawer in such a way that the $(d-2)$-dimensional bottom of $H$ is packed into the $(d-2)$-dimensional bottom of the drawer by using the $D_{1}(d-2)$-algorithm.

- Otherwise we close the active bin and open a new active bin to pack $H$.

Three examples presented below illustrate the packing method.

Example $8 \mathrm{~A}$ list of 3 -dimensional boxes $H_{1}=(1 / 7+\epsilon, 1 / 14+\epsilon, 1 / 7+\epsilon)$, $H_{2}=(1, \epsilon, 1 / 7+\epsilon), H_{3}=(1 / 7+2 \epsilon, 1 / 14+2 \epsilon, 1 / 7+2 \epsilon), H_{4}=(1,2 \epsilon, 1 / 7+$ $2 \epsilon), \ldots, H_{240}$, for sufficiently small $\epsilon>0$ is packed as shown on Figs. 15 and 16 (on these figures $\epsilon$ equals $1 / 70$ ).

The front wall of $H_{1}$ is a rectangle $(1 / 14+\epsilon) \times(1 / 7+\epsilon)$ and $F_{1}=P\left(H_{1}\right)=$ $1 / 7 \times 2 / 7$ is the smallest $\lambda$-rectangle containing this rectangle. We open a drawer with the front wall $F_{1}$, i.e., the first basic drawer. $H_{1}$ is packed into this drawer in the place $[0,1 / 7+\epsilon] \times[0,1 / 14+\epsilon] \times[0,1 / 7+\epsilon]$.

Let $j$ be the greatest integer such that $\epsilon \leq \lambda / 2^{j}$ and let $\zeta=\lambda / 2^{j}$. The front wall of $H_{2}$ is a rectangle $\epsilon \times(1 / 7+\epsilon)$ and $F_{2}=P\left(H_{2}\right)=\zeta \times 2 / 7$ is the smallest 


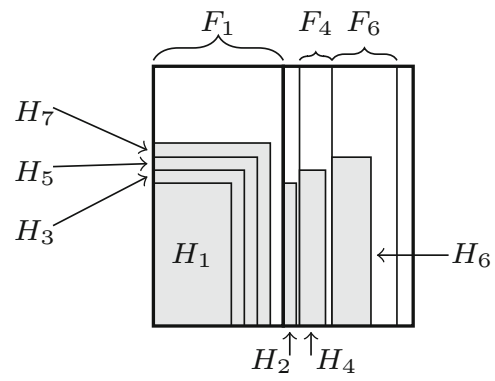

first two basic units of $B_{1}$

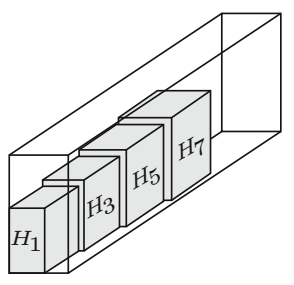

first drawer

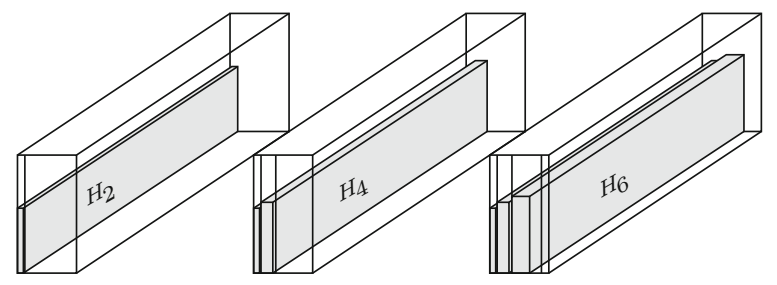

(b)

(c)

Fig. 15 Example 8, first drawer after packing $H_{1}, H_{3}, H_{5}$ and $H_{7}$ and next three drawers created in the second basic drawer for packing: a $\mathrm{H}_{2}, \mathbf{b} \mathrm{H}_{4}, \mathbf{c} \mathrm{H}_{6}$

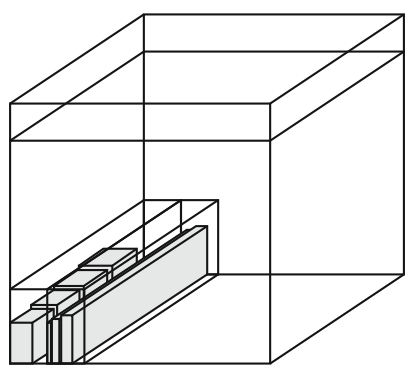

Fig. 16 Example 8, the bin

$\lambda$-rectangle containing this rectangle. Since there is no open drawer with front wall $F_{2}$, we open a new drawer $[0,1] \times[1 / 7,1 / 7+\zeta] \times[0,2 / 7]$ and pack $H_{2}$ in the place $[0,1] \times[1 / 7,1 / 7+\epsilon] \times[0,1 / 7+\epsilon]$.

$H_{3}$ is packed similarly to $H_{1}$. The front wall of $H_{3}$ is a rectangle $(1 / 14+2 \epsilon) \times$ $(1 / 7+2 \epsilon)$ and $F_{3}=P\left(H_{3}\right)=1 / 7 \times 2 / 7$. There is an open drawer with the front wall $F_{3}=F_{1}$ (the first basic unit) with enough empty space, so we pack $H_{3}$ in the place $[1 / 7+\epsilon, 2 / 7+3 \epsilon] \times[0,1 / 14+2 \epsilon] \times[0,1 / 7+2 \epsilon]$.

The front wall of $H_{4}$ is a rectangle $2 \epsilon \times(1 / 7+2 \epsilon)$ and $F_{4}=P\left(H_{4}\right)=2 \zeta \times 2 / 7$ is the smallest $\lambda$-rectangle containing this rectangle. There is no open drawer with front wall $F_{4}$, therefore we open such drawer $[0,1] \times[1 / 7+\zeta, 1 / 7+3 \zeta] \times[0,2 / 7]$ and pack $H_{4}$ in the place $[0,1] \times[1 / 7+\zeta, 1 / 7+\zeta+2 \epsilon] \times[0,1 / 7+\epsilon]$. We continue to pack odd items into the first drawer as long as it is possible. Then a new drawer will be opened for them. Each even item is packed into an individual drawer of height $\lambda$. 


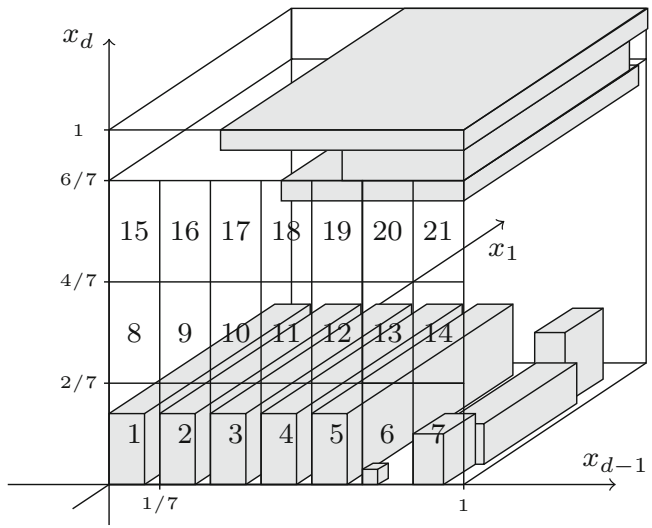

Fig. 17 Drawers in $B_{1}(d), d=3$

Note that $\epsilon$ is relatively large on Fig. 15. However, if $\epsilon$ is sufficiently small, for example smaller than $10^{-6}$, then $H_{2}, H_{4}, \ldots, H_{240}$ are packed into individual drawers of height $\lambda$ created in the second basic drawer. Six items $H_{1}, H_{3}, H_{5}, H_{7}, H_{9}, H_{11}$ are packed into the first basic drawer. Moreover, we pack six items $H_{12 k-23}, H_{12 k-21}, \ldots$, $H_{12 k-13}$ into the $k$ th basic drawer, for $k=3,4, \ldots, 21$. Clearly, $H_{241}=H_{12 \cdot 22-23}$ is the first item that cannot be packed into the active bin by $D_{1}(d)$-algorithm.

Example 9 The following example is a precise description of the packed items showed on Fig. 17. Let $d=3$ and let $H_{1}=\cdots=H_{5}=0.9 \times 0.1 \times 0.2, H_{6}=0.05 \times 0.05 \times$ $0.05, H_{7}=0.17 \times 0.1 \times 0.17, H_{8}=0.49 \times 0.13 \times 0.13, H_{9}=0.2 \times 0.1 \times 0.23$, $H_{10}=1 \times 0.6 \times 0.05, H_{11}=0.9 \times 0.34 \times 0.08$ and $H_{12}=0.95 \times 0.51 \times 0.05$.

For $i=1, \ldots, 5$, each item $H_{i}$ is packed into the $(0,1)$-drawer (i.e., the basic drawer) number $i$ in the place $[0,0.9] \times[(i-1) / 7,(i-1) / 7+0.1] \times[0,0.2]$. The item $H_{6}$ is packed into the drawer $1 \times 1 / 14 \times 1 / 14$ with the front wall contained in the unit number 6 in the place: $[0,0.05] \times[5 / 7,5 / 7+0.05] \times[0,0.05], H_{7}$ is packed into the drawer with the front wall contained in the unit number 7 so that its $(d-2)$-dimensional bottom (the segment of length 0.17$)$ is packed into the $(d-2)$-dimensional bottom of the drawer (the segment of length 1 ) by the $D_{1}$ (1) method, i.e., we pack $H_{7}$ in the place $[0,0.17] \times[6 / 7,6 / 7+0.1] \times[0,0.17]$. Since $P\left(H_{8}\right) \neq P\left(H_{6}\right)$, the item $H_{8}$ cannot be packed in one drawer with $H_{6}$. Notice that $P\left(H_{8}\right)=P\left(H_{7}\right)$ as well as $P\left(H_{9}\right)=$ $P\left(H_{7}\right)$ and since there is enough space in drawer with the front wall contained in the unit number 7 we do not open a new drawer for $H_{8}$ or $H_{9}$. According to the Next Fit algorithm $H_{8}$ is packed in $[0.17,0.17+0.49] \times[6 / 7,6 / 7+0.13] \times[0,0.13]$ and $H_{9}$ in $[0.66,0.66+0.2] \times[6 / 7,6 / 7+0.1] \times[0,0.23]$. The big item $H_{10}$ is packed in $[0,1] \times[1-0.6,1] \times[1-0.05,1]$ (along the right edge of the bin, i.e., the segment with endpoints $(0,1,0.95)$ and $(0,1,1)$ is contained in this box $), H_{11}$ in $[0,0.9] \times[1-0.34,1] \times[0.95-0.08,0.95]$ and $H_{12}$ in $[0,0.95] \times[1-0.51,1] \times$ $[0.87-0.05,0.87]$. 
Example 10 Assume that $d=5$ and that the $(d-2)$-dimensional bottoms of hyperboxes $U_{i}$ (for $i=1, \ldots 12$ ) are boxes $H_{i}$ described in Example 9. Let the length of the 4 th and the 5 th edge of each $U_{i}$ be equal to 0.01 , i.e., $U_{1}=0.9 \times 0.1 \times 0.2 \times$ $0.01 \times 0.01, \ldots, U_{12}=0.95 \times 0.51 \times 0.05 \times 0.01 \times 0.01$.

Since $0.01<2 / 7$ all these hyperboxes are small items. Let $U_{13}$ be a big item of the size $0.4 \times 0.4 \times 0.4 \times 0.4 \times 0.1$.

All small items $U_{1}, \ldots, U_{12}$ are packed into the first drawer $1 \times 1 \times 1 \times 1 / 56 \times$ $1 / 56$ with the front wall contained in the first unit so that the $(d-2)$-dimensional bottoms of $U_{i}$ (the boxes $H_{i}$ ) are packed into the $(d-2)$-dimensional bottom of the drawer (the unit cube) as in Example 9. For instance, $U_{8}$ is packed in the place $[0.17,0.66] \times[6 / 7,6 / 7+0.13] \times[0,0.13] \times[0,0.01] \times[0,0.01], U_{11}$ is packed in $[0,0.9] \times[0.66,1] \times[0.87,0.95] \times[0,0.01] \times[0,0.01]$. The only big item $U_{13}$ is packed in $[0,0.4] \times[0,0.4] \times[0,0.4] \times[0.6,1] \times[0.9,1]$ along the right edge of the bin, i.e., the segment with endpoints $(0,0,0,1,0.9)$ and $(0,0,0,1,1)$ is contained in $U_{13}$.

Theorem 1 The asymptotic competitive ratio for the $D_{1}(d)$-algorithm is not greater than $3.5^{d}$.

Proof We show that the average occupation in each bin is greater than

$$
\sigma_{d}=(2 / 7)^{d}
$$

The proof is inductive.

For $d=1$ the average occupation in each bin is greater than $1 / 2>2 / 7$, for $d=2$ (see Zhang et al. 2014) it is not smaller than $0.197>(2 / 7)^{2}$.

Let $d \geq 3$. Assume that the statement holds in each dimension $n \in\{1,2, \ldots, d-$ $1\}$.

Denote by $\sigma(l)$ the total volume of items packed into the $l$ th bin $\mathcal{B}_{l}$. To prove that the average occupation in each bin is greater than $\sigma_{d}$ it suffices to show that either $\sigma(l)>\sigma_{d}$ or $\sigma(l)$ plus the volume of the first item that cannot be packed into $\mathcal{B}_{l}$ is greater than $2 \sigma_{d}$ (consequently, $\sigma(l)+\sigma(l+1)>2 \sigma_{d}$ ).

Consider a few cases depending on the size of the first hyperbox item

$$
H_{u}=u_{1} \times \cdots \times u_{d-1} \times u_{d}
$$

where $u_{1} \geq \cdots \geq u_{d-2} \geq u_{d} \geq u_{d-1}$, that cannot be packed into $\mathcal{B}_{l}$. Denote by $h$ the sum of heights of big items packed into $\mathcal{B}_{l}$ and let $h_{u}=u_{d-1}$.

By the inductive assumption (for $n=d-2$ ) and by the fact that the smallest $\lambda$-rectangle containing a rectangle $R=a_{d-1} \times a_{d}$ is of area smaller than 4 times the area of $R$, the average occupation in any closed $(i, j)$-drawer $\Lambda_{i, j}$ is greater than

$$
\frac{1}{4} \cdot \sigma_{d-2} \cdot \operatorname{vol}\left(\Lambda_{i, j}\right)
$$

where $\operatorname{vol}(D)$ denotes the $d$-dimensional volume of a drawer $D$. The total volume of items packed in any open drawer can be close to 0 . There is at most one open 
$(i, j)$-drawer for any integers $i$ and $j$. The total volume of open drawers of height $\lambda$ is smaller than $\lambda \cdot \lambda+\frac{1}{2} \lambda \cdot \lambda+\frac{1}{4} \lambda \cdot \lambda+\cdots=2 \lambda^{2}$. The sum of volumes of open drawers of height $\lambda / 2$ is smaller than $\frac{1}{2} \lambda \cdot \frac{1}{2} \lambda+\frac{1}{4} \lambda \cdot \frac{1}{2} \lambda+\cdots=\frac{1}{2} \lambda^{2}$. The total volume of open drawers of height $\lambda / 4$ is smaller than $\frac{1}{4} \lambda \cdot \frac{1}{4} \lambda+\frac{1}{8} \lambda \cdot \frac{1}{4} \lambda+\cdots=\frac{1}{8} \lambda^{2}$ and so on. Consequently, the sum of volumes of open drawers is smaller than

$$
2 \lambda^{2}+\frac{1}{2} \lambda^{2}+\frac{1}{8} \lambda^{2}+\cdots=\frac{8}{3} \lambda^{2}
$$

Denote by $k \in\{1, \ldots, 21\}$ the greatest integer such that the interior of a drawer with the front wall contained in the $k$ th basic unit has a non-empty intersection with a packed small item.

Case $1 H_{u}$ is small and $h \leq 1 / 7$.

For a moment we forget about drawers and consider only two-dimensional packing. We count the sum of areas of $\lambda$-rectangles being the front walls of all open and closed drawers. Let $F_{u}=P\left(H_{u}\right)$ be the smallest $\lambda$-rectangle containing the front wall of $H_{u}$ (i.e, containing the rectangle $u_{d-1} \times u_{d}$ ). Clearly, $F_{u}$ cannot be packed into $B_{1}$ by $\Lambda_{1}$-method. If $F_{u}$ is $\lambda$-max, then by Lemma 2 the empty space in $B_{1}$ is smaller than $8 \lambda^{2} / 3$. Otherwise, $k=21$. If a $\lambda$-rectangle of width smaller than $\lambda$ was packed in the unit number 21 , then by Lemma 1 the empty space in $B_{1}$ is smaller than $7 \lambda^{2} / 6$. If a rectangle $\lambda$-max was packed in the union of units with number 20 and 21 , then by Lemma 2 the empty space in $B_{1}$ is smaller than $13 \lambda^{2} / 6$. This means that the total area of $\lambda$-rectangles packed into $B_{1}$, i.e., the sum of areas of front walls of all open and closed drawers, is greater than $21 \cdot \frac{1}{2} \lambda^{2}-\frac{8}{3} \lambda^{2}=\frac{47}{6} \lambda^{2}$.

Consequently, the total volume of all closed and open drawers is greater than $\frac{47}{6} \lambda^{2}$. Since the sum of volumes of open drawers is smaller than $8 \lambda^{2} / 3$, it follows that the total volume of closed drawers is greater than $\frac{47}{6} \lambda^{2}-\frac{8}{3} \lambda^{2}=\frac{31}{6} \lambda^{2}$. Hence, total volume of small items packed into $\mathcal{B}_{l}$ is greater than

$$
\frac{1}{4} \cdot \sigma_{d-2} \cdot \frac{31}{6} \lambda^{2}=\frac{1}{4} \cdot\left(\frac{2}{7}\right)^{d-2} \cdot \frac{31}{6} \cdot\left(\frac{2}{7}\right)^{2}>\left(\frac{2}{7}\right)^{d}=\sigma_{d}
$$

Case $2 H_{u}$ is small and $1 / 7<h<2 / 7$. Clearly, $k \geq 13$.

The total volume of big items packed into $\mathcal{B}_{l}$ is greater than $h \cdot(2 / 7)^{d-1}$. The total volume of open and closed drawers, by Lemma 2 , is greater than $13 \cdot \frac{1}{2} \lambda^{2}-\frac{5}{3} \lambda^{2}=\frac{29}{6} \lambda^{2}$. The total volume of closed drawers is greater than $\frac{29}{6} \lambda^{2}-\frac{8}{3} \lambda^{2}=\frac{13}{6} \lambda^{2}$. Consequently,

$$
\sigma(l) \geq \frac{1}{4} \cdot \sigma_{d-2} \cdot \frac{13}{6} \lambda^{2}+h \cdot\left(\frac{2}{7}\right)^{d-1}>\left(\frac{13}{24}+\frac{1}{7} \cdot \frac{7}{2}\right) \cdot\left(\frac{2}{7}\right)^{d}>\sigma_{d} .
$$

Case $3 h \geq 2 / 7$. The total volume of big items packed into $\mathcal{B}_{l}$ is greater than

$$
h \cdot(2 / 7)^{d-1} \geq(2 / 7)^{d}
$$

In the next cases we will assume that $h<2 / 7$ and that $H_{u}$ is big. 


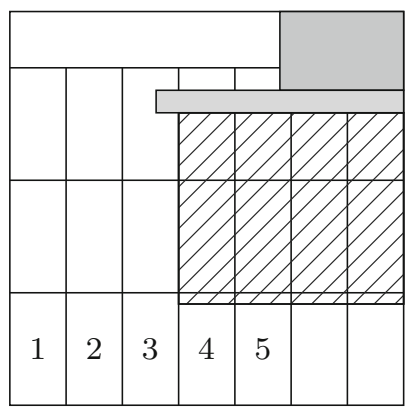

Fig. 18 Case 4

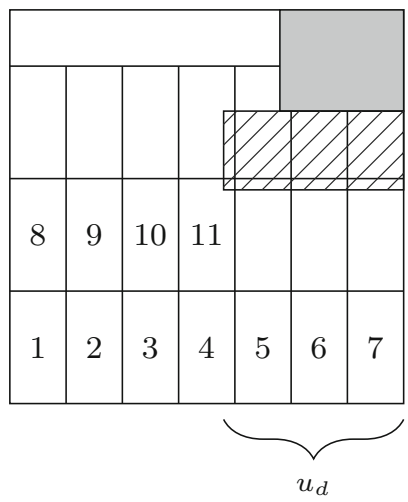

Fig. 19 Case $5, k=11$

Case $4 H_{u}$ is big and $k \in\{1, \ldots, 7\}$. Since $h<2 / 7$ and $h+h_{u}>5 / 7$ (see Fig. 18 , where the front wall of the active bin and front walls of big items are shown), it follows that $h_{u}>3 / 7$ and

$$
\sigma(l)+\sigma(l+1) \geq \operatorname{vol}\left(H_{u}\right)>\left(\frac{3}{7}\right)^{d}>2 \cdot\left(\frac{2}{7}\right)^{d}=2 \sigma_{d}
$$

Case $5 H_{u}$ is big and $k \in\{8, \ldots, 11\}$. If $h+h_{u}>5 / 7$, then we proceed as in Case 4. Otherwise, $u_{d}>3 / 7$ and $h+h_{u}>3 / 7$ (see Fig. 19). Since $h<2 / 7$, it follows that

$$
\begin{aligned}
\sigma(l)+\operatorname{vol}\left(H_{u}\right) & >h\left(\frac{2}{7}\right)^{d-1}+h_{u} \cdot\left(u_{d}\right)^{d-1}>h\left(\frac{2}{7}\right)^{d-1}+\left(\frac{3}{7}-h\right) \cdot\left(\frac{3}{7}\right)^{d-1} \\
& =\left[h+\left(\frac{3}{7}-h\right)\left(\frac{3}{2}\right)^{d-1}\right] \cdot\left(\frac{2}{7}\right)^{d-1} \\
& \geq\left[h+\left(\frac{3}{7}-h\right)\left(\frac{3}{2}\right)^{2}\right] \cdot\left(\frac{2}{7}\right)^{d-1} \\
& >\left[\frac{2}{7}+\left(\frac{3}{7}-\frac{2}{7}\right) \cdot \frac{9}{4}\right] \cdot \frac{7}{2} \cdot\left(\frac{2}{7}\right)^{d}=\frac{17}{8} \cdot\left(\frac{2}{7}\right)^{d}>2 \sigma_{d} .
\end{aligned}
$$




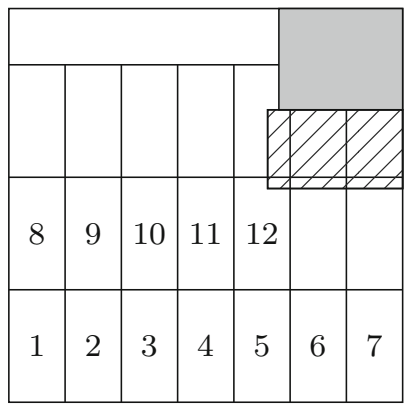

Fig. 20 Case $6, k=12$

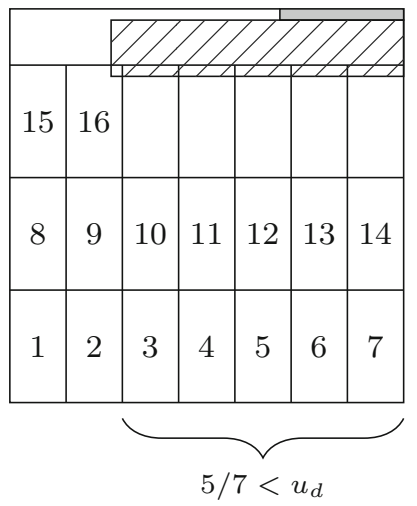

Fig. 21 Case 7, $k=16$

Case $6 H_{u}$ is big and $k \in\{12,13,14\}$. If $k=12$, then the total volume of small items in $\mathcal{B}_{l}$ is, by Lemma 2, greater than

$$
\left(12 \cdot \frac{1}{2} \lambda^{2}-\frac{7}{6} \lambda^{2}-\frac{8}{3} \lambda^{2}\right) \cdot \frac{1}{4} \sigma_{d-2}=\frac{13}{24}\left(\frac{2}{7}\right)^{d} .
$$

If $k \in\{13,14\}$, then the total volume of small items in $\mathcal{B}_{l}$ is greater than

$$
\left(13 \cdot \frac{1}{2} \lambda^{2}-\frac{5}{3} \lambda^{2}-\frac{8}{3} \lambda^{2}\right) \cdot \frac{1}{4} \sigma_{d-2}=\frac{13}{24}\left(\frac{2}{7}\right)^{d}
$$

Since $h+h_{u}>3 / 7$ (see Fig. 20),

$$
\sigma(l)+\operatorname{vol}\left(H_{u}\right) \geq \frac{13}{24} \cdot\left(\frac{2}{7}\right)^{d}+\frac{3}{7} \cdot\left(\frac{2}{7}\right)^{d-1}>2 \cdot\left(\frac{2}{7}\right)^{d} .
$$

Case $7 H_{u}$ is big and $k \in\{15,16\}$. In the case when $k=15$, the total volume of small items in $\mathcal{B}_{l}$ is by Lemma 1 greater than

$$
\left(15 \cdot \frac{1}{2} \lambda^{2}-\frac{7}{6} \lambda^{2}-\frac{8}{3} \lambda^{2}\right) \cdot \frac{1}{4} \sigma_{d-2}=\frac{11}{12}\left(\frac{2}{7}\right)^{d}>\frac{19}{24}\left(\frac{2}{7}\right)^{d} \text {. }
$$


In the case when $k=16$, the total volume of small items in $\mathcal{B}_{l}$ is, by Lemma 2 , greater than

$$
\left(16 \cdot \frac{1}{2} \lambda^{2}-\frac{13}{6} \lambda^{2}-\frac{8}{3} \lambda^{2}\right) \cdot \frac{1}{4} \sigma_{d-2}=\frac{19}{24}\left(\frac{2}{7}\right)^{d} .
$$

If $h \geq 5 / 84$, then

$$
\sigma(l)>\frac{19}{24}\left(\frac{2}{7}\right)^{d}+\frac{5}{84} \cdot\left(\frac{2}{7}\right)^{d-1}=\sigma_{d}
$$

If $h+h_{u}>3 / 7$, we proceed as in Case 6. If $h+h_{u} \leq 3 / 7$ and $h<5 / 84$, then

$$
h_{u}>1 / 7-5 / 84=1 / 12
$$

and $u_{d}>5 / 7$ (see Fig. 21). This implies that

$$
\sigma(l)+\operatorname{vol}\left(H_{u}\right)>\frac{19}{24}\left(\frac{2}{7}\right)^{d}+\frac{1}{12} \cdot\left(\frac{5}{7}\right)^{d-1} .
$$

It is easy to check that

$$
\left(\frac{5}{7}\right)^{d-1}>\frac{29}{2}\left(\frac{2}{7}\right)^{d}
$$

for $d \geq 3$. Consequently,

$$
\sigma(l)+\operatorname{vol}\left(H_{u}\right)>\frac{19}{24}\left(\frac{2}{7}\right)^{d}+\frac{1}{12} \cdot \frac{29}{2}\left(\frac{2}{7}\right)^{d}=2\left(\frac{2}{7}\right)^{d} .
$$

Case $8 H_{u}$ is big and $k \geq 17$. According to Lemma 2 , if $k=17$, then the total volume of small items in $\mathcal{B}_{l}$ is greater than

$$
\left(17 \cdot \frac{1}{2} \lambda^{2}-\frac{7}{6} \lambda^{2}-\frac{8}{3} \lambda^{2}\right) \cdot \frac{1}{4} \sigma_{d-2}=\frac{7}{6}\left(\frac{2}{7}\right)^{d}>\sigma_{d} .
$$

If $k=18$, then the total volume of small items in $\mathcal{B}_{l}$ is greater than

$$
\left(18 \cdot \frac{1}{2} \lambda^{2}-\frac{13}{6} \lambda^{2}-\frac{8}{3} \lambda^{2}\right) \cdot \frac{1}{4} \sigma_{d-2}=\frac{25}{24}\left(\frac{2}{7}\right)^{d}>\sigma_{d} .
$$

If $k \geq 19$, then the total volume of small items in $\mathcal{B}_{l}$ is greater than

$$
\left(19 \cdot \frac{1}{2} \lambda^{2}-\frac{8}{3} \lambda^{2}-\frac{8}{3} \lambda^{2}\right) \cdot \frac{1}{4} \sigma_{d-2}=\frac{25}{24}\left(\frac{2}{7}\right)^{d}>\sigma_{d}
$$

The average occupation in each bin is greater than $(2 / 7)^{d}$. Consequently, the asymptotic competitive ratio of this packing strategy is not greater than

$$
\left[(2 / 7)^{d}\right]^{-1}=3.5^{d} \text {. }
$$



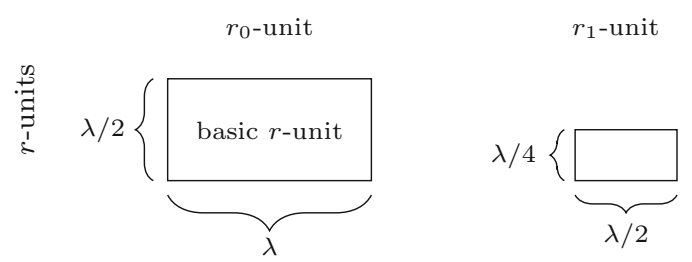

$r_{2}$-unit $\quad r_{3}$-unit
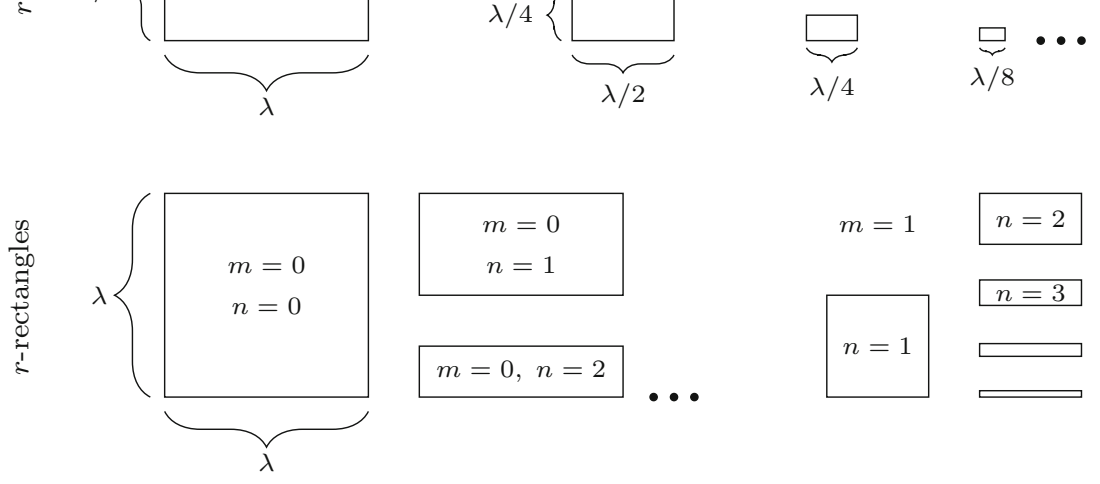

Fig. $22 r$-units and $r$-rectangles

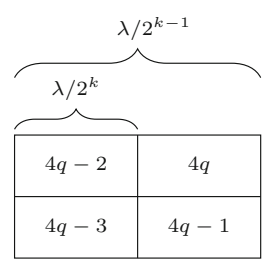

Fig. 23 The division of an $r_{k-1}$-unit into four $r_{k}$-units

\section{$5 \Lambda_{2}$-method for packing $r$-rectangles}

Let $\lambda>0$ and let $k$ be a non-negative integer. An $r_{k}$-unit is a rectangle of width $\lambda / 2^{k}$ and height $\lambda / 2^{k+1}$ (see Fig. 22). A basic r-unit is $r_{0}$-unit. Of course, an $r_{k}$-unit is the image of a $\lambda_{k}$-unit in rotation of $90^{\circ}$. In Sect. 3 , basic units were divided into smaller units, and here basic $r$-units will be divided into smaller $r$-units in the same way (see Fig. 23).

Let $r$-rectangle be a rectangle of width $\lambda / 2^{m}$ and height $\lambda / 2^{n}$ for some $0 \leq m \leq n$. Clearly, each $r$-rectangle is the image of a $\lambda$-rectangle in rotation of $90^{\circ}$.

Let $U$ be the union of $k$ basic r-units with pairwise disjoint interiors numbered with natural numbers from 1 to $n$. When an $r_{k-1}$-unit $(k \geq 1)$ numbered with $q$ is divided into four $r_{k}$-units, these $r_{k}$-units are numbered from $4 q-3$ to $4 q$ as on Fig. 23.

Consider a sequence of $r$-rectangles $R_{1}, R_{2}, \ldots$ of widths not greater than $\lambda / 2$. Note than neither $\lambda \times \lambda$ nor $\lambda \times \frac{1}{2} \lambda$ occurs in the sequence.

We will use the analogue of the $\Lambda_{1}$-method of packing (see Fig. 24).

\section{$\Lambda_{2}$-method of packing an $r$-rectangle of width $l_{i}=\lambda / 2^{i}$ into $U$}

1. If an $r$-rectangle is not a square, then it is packed as low as possible into the lowest indexed $r_{i}$-unit and, obviously, with enough empty space. 

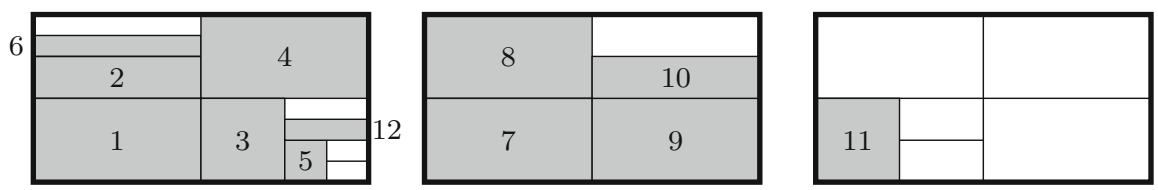

Fig. 24 Packing items into three basic $r$-units by $\Lambda_{2}$-method. Numbers indicate the order in which the items arrive

If there is no such unit, find the greatest $k \leq i$ such that there is an empty $r_{k}$-unit. From among empty $r_{k}$-units choose the lowest indexed one and then divide it into four $r_{k+1}$-units. If $r_{k+1}>r_{i}$, then the lowest indexed $r_{k+1}$-unit is again divided into four smaller units. The division is repeated until an $r_{i}$-unit is created. Now the $r$-rectangle is packed into the lowest indexed $r_{i}$-unit as low as possible.

2. An $r$-rectangle that is a square of side length $l_{i}=\lambda / 2^{i}, i \geq 1$, is packed, if possible, into two empty $r_{i}$-units, that were created through the division of one $r_{i-1}$-unit. If there are no such units the division of a bigger unit is conducted as described in the previous case. Finally the $r$-rectangle is packed into two lowest indexed $r$-units.

Example 11 Figure 24 illustrates $\Lambda_{2}$-method. The method is very similar to $\Lambda_{1}$-method shown in Example 7. The main difference between the two methods is that $\Lambda_{1}$ packs as much to the left as possible, while $\Lambda_{2}$ puts items as low as it is possible.

The first $r$-rectangle, by Rule 1 , should be packed into an $r_{1}$-unit. Since there is no such unit, the first $r_{0}$-unit is divided and the $r$-rectangle is then packed into the lowest indexed $r_{1}$-unit. The second item is packed, by Rule 1, into the lowest indexed (the second) $r_{1}$-unit as low as possible. The third item is a square thus we use Rule 2 and divide the lowest indexed $r_{1}$-unit. The third $r$-rectangle is packed into two lowest indexed $r_{2}$-units. The fourth item is packed, by Rule 1 , into an $r_{1}$-unit with enough empty space. To pack the fifth $r$-rectangle, we need to perform another division and the item is packed, by Rule 2, into two $r_{3}$-units. Items from 6 to 10 are all packed by Rule 1 into lowest indexed $r_{1}$-units with enough empty space as low as possible. The eleventh item is a square and thus is packed by using Rule 2: the third basic $r$-unit is partitioned and then the lowest indexed $r_{1}$-unit is divided again. The eleventh item is packed into two lowest indexed $r_{2}$-units. We use Rule 1 for the twelfth $r$-rectangle which can be fitted into an $r_{2}$-unit in the first basic $r$-unit.

Lemma 3 Assume that a sequence of $r$-rectangles of width not greater than $\lambda / 2$ was packed into $U$ by $\Lambda_{2}$-method. Let $W \subset U$ be the union of basic r-units into which an $r$-rectangle was packed. The empty space in $W$ is smaller than $\eta=\frac{2}{3} \lambda^{2}$.

Proof We follow the same steps as in the proof of Lemma 1. However, now there are no $r$-rectangles of width $\lambda$ in the sequence.

First, we calculate the empty space in r-units that are partially packed. Using the same arguments, we get that the empty space in all r-units of width $\lambda_{i}=\lambda / 2^{i}$ is not greater than $\frac{1}{2} \lambda_{i}^{2}$. Since the sequence of items to pack consists of $r$-rectangles of width smaller than $\lambda$, there are no partially packed basic $r$-units (of area $\lambda^{2} / 2$ ). Consequently, 


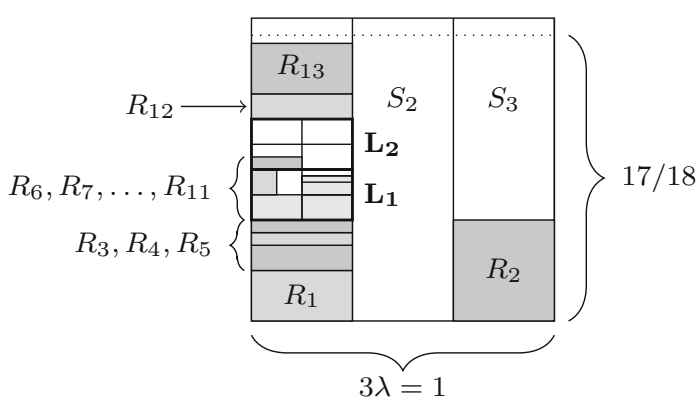

Fig. $25 \Lambda_{2}^{+}$-method

the empty space in partially packed $r$-units is not greater than

$$
\sum_{i \geq 1} \frac{1}{2} \lambda_{i}^{2}=\sum_{i \geq 1} \frac{1}{2}\left(\frac{1}{2^{i}} \lambda\right)^{2}=\frac{1}{2} \lambda^{2} \sum_{i \geq 1} \frac{1}{4^{i}}=\frac{1}{6} \lambda^{2} .
$$

Note that in the proof of Lemma 1 the empty space in partially packed $\lambda$-units was not greater than $2 \lambda^{2} / 3$; now in $r$-units it is not greater than $2 \lambda^{2} / 3-\lambda^{2} / 2=\lambda^{2} / 6$.

The number of empty r-units in $\Lambda_{2}$-method is the same as in $\Lambda_{1}$-method, thus the empty space in empty r-units is not greater than $\frac{1}{2} \lambda^{2}$.

Finally, the empty space in $W$ is less than

$$
\frac{1}{6} \lambda^{2}+\frac{1}{2} \lambda^{2}=\frac{2}{3} \lambda^{2}
$$

Let

$$
\begin{aligned}
\lambda & =1 / 3, \\
B_{2} & =[0,1] \times[0,1]
\end{aligned}
$$

and let $R_{1}, R_{2}, \ldots$ be a sequence of $r$-rectangles. $B_{2}$ is partitioned into three rectangles $S_{w}=[(w-1) / 3, w / 3] \times[0,1]$ for $w=1,2,3$.

As in Sect. 4 the value of $\lambda$ is chosen to balance the average ratios of packing of big and small items. Exactly the same reasoning is behind the choice of the height 17/18 of packing in $S_{1}, S_{2}, S_{3}$.

We give an outline of the $\Lambda_{2}^{+}$-packing method presented below. All $r$-rectangles are packed from bottom to top.

- Squares $1 / 3 \times 1 / 3$ are packed first into $S_{3}$ up to the height $17 / 18$, then into $S_{2}$ up to the height $17 / 18$ (see $R_{2}$ on Fig. 25).

- $r$-rectangles of width smaller than $1 / 3$ are packed into basic $r$-units $1 / 3 \times 1 / 6$, called containers (see $L_{1}$ and $L_{2}$ on Fig. 25), by using the $\Lambda_{2}$-method (see $R_{6}, R_{7}, \ldots, R_{11}$ on Fig. 25). 
- Containers (see $L_{1}$ and $L_{2}$ on Fig. 25) are created and other items of width $1 / 3$ that are not squares (as $R_{1}, R_{3}, R_{4}, R_{5}, R_{12}, R_{13}$ on Fig. 25) are packed first into $S_{1}$ up to the height $17 / 18$, then into $S_{2}$ up to the height $17 / 18$, then into $S_{3}$.

- If an $r$-rectangle cannot be packed or a container cannot be created under the height $17 / 18$, it is packed or created in $B_{2}$ as low as possible.

Let $P$ be the topmost $r$-rectangle preceding $R_{i}$ packed in $S_{w}$. Denote by $b_{w}(i)$ the distance between the bottom of $S_{w}$ and

- the top of $P$, provided the width of $P$ is $1 / 3$;

- the top of the container into which $P$ was packed, provided the width of $P$ is smaller than $1 / 3$.

Moreover, $b_{\max }(i)=\max \left[b_{1}(i), b_{2}(i), b_{3}(i)\right], b_{\min }(i)=\min \left[b_{1}(i), b_{2}(i), b_{3}(i)\right]$. For example, on Fig. 25, $b_{1}(1)=b_{2}(1)=b_{3}(1)=0, b_{1}(2)=b_{1}(3)=1 / 6, b_{3}(3)=$ $1 / 3, b_{1}(4)=1 / 4, b_{1}(5)=7 / 24, b_{1}(6)=1 / 3, b_{1}(7)=\cdots=b_{1}(11)=1 / 2$, $b_{1}(12)=2 / 3$.

\section{$\Lambda_{2}^{+}$-method of packing of an r-rectangle $R_{i}$ into $B_{2}$}

1. Any item that is packed or any container that is created is placed in a bin in such a way that its interior is disjoint with any $r$-rectangle packed so far and with any container created earlier.

2. If $R_{i}$ is a square $1 / 3 \times 1 / 3$, then

(a) if $b_{3}(i) \leq 17 / 18-1 / 3=11 / 18$, then $R_{i}$ is packed into $S_{3}$ as low as it is possible, (see the two squares on the right on Fig. 31);

(b) if $b_{3}(i)>11 / 18$ and $b_{2}(i) \leq 11 / 18$, then $R_{i}$ is packed into $S_{2}$ as low as it is possible, (see the middle square $1 / 3 \times 1 / 3$ on Fig. 31 ; now $b_{3}(i)=2 / 3>$ $11 / 18$ and $\left.b_{2}(i)=11 / 18\right)$;

(c) otherwise $R_{i}$ is packed into $S_{q}$ as low as it is possible, where $q \in\{1,2,3\}$ is an integer such that $b_{q}(i)=b_{\min }(i)$. If $b_{\min }(i)$ is not unique then $R_{i}$ is packed into the lowest indexed $b_{q}(i)=b_{\min }(i)$.

3. If $R_{i}$ is a rectangle of width $w_{i}=1 / 3$ and height $t_{i} \leq 1 / 6$, then

(a) if $b_{1}(i) \leq 17 / 18-t_{i}$, then $R_{i}$ is packed into $S_{1}$ as low as it is possible, (see $R_{1}, R_{3}, R_{4}, R_{5}, R_{12}, R_{13}$ on Fig. 25);

(b) if $b_{1}(i)>17 / 18-t_{i}$ and $b_{2}(i) \leq 17 / 18-t_{i}$, then $R_{i}$ is packed into $S_{2}$ as low as it is possible, (see the first $r$-rectangle packed in $S_{2}$ on Fig. 29);

(c) if $b_{1}(i)>17 / 18-t_{i}, b_{2}(i)>17 / 18-t_{i}$ and $b_{3}(i) \leq 17 / 18-t_{i}$, then $R_{i}$ is packed into $S_{3}$ as low as it is possible (see the third, i.e., the topmost $r$-rectangle packed in $S_{3}$ on Fig. 29);

(d) otherwise, $R_{i}$ is packed into $S_{q}$ as low as it is possible, where $q \in\{1,2,3\}$ is an integer such that $b_{q}(i)=b_{\min }(i)$ (see the patterned $r$-rectangle that we try to pack in $S_{2}$ on Fig. 29). If $b_{\min }(i)$ is not unique then $R_{i}$ is packed into the lowest indexed $b_{q}(i)=b_{\min }(i)$.

4. If $w_{i} \leq 1 / 6$, then let $U$ be the union of created containers contained in $B_{2}$. If $U=\emptyset$, then $k=0$. Otherwise denote by $k$ the number of created containers. These containers are numbered from 1 to $k$. 
(a) if $R_{i}$ can be packed into $U$ by $\Lambda_{2}$-method, then we do it $\left(R_{7}, \ldots, R_{10}\right.$ on Fig. 25 are packed into the existing container $L_{1}$ );

(b) otherwise we open a new container of number $k+1$ as follows:

- if $b_{1}(i) \leq 17 / 18-1 / 6=7 / 9$, then $L$ is a basic r-unit packed into $S_{1}$ as low as it is possible,

For example, on Fig. 25, if $i=6$, then there is no created container; the new container $L_{1}$ is opened ( $R_{6}$ is the first item packed into a freshly created container) in such a place into which a basic $r$-unit should be packed, i.e., in $S_{1}$ as low as it is possible; if $i=11$, then $R_{11}$ cannot be packed in $L_{1}$; therefore we create a new container $L_{2}$ (to pack this rectangle) in such a place into which a basic $r$-unit should be packed, i.e., in $S_{1}$ as low as it is possible;

- if $b_{1}(i)>7 / 9$ and $b_{2}(i) \leq 7 / 9$, then $L$ is a basic r-unit packed into $S_{2}$ as low as it is possible;

- if $b_{1}(i)>7 / 9, b_{2}(i)>7 / 9$ and $b_{3}(i) \leq 7 / 9$, then $L$ is a basic r-unit packed into $S_{3}$ as low as it is possible;

- otherwise, $L$ is a basic r-unit packed into $S_{q}$ as low as it is possible, where $b_{q}(i)=b_{\min }(i)$;

$R_{i}$ is packed into $U \cup L$ by $\Lambda_{2}$-method.

\section{Second drawer algorithm}

In this section a drawer algorithm $D_{2}(d)$ with the asymptotic competitive ratio not greater than $12 \cdot 3^{d}$ is presented.

Let $\lambda=1 / 3$. Each hyperbox $H$ is rotated to satisfy $a_{1} \geq \cdots \geq a_{d-1} \geq a_{d}$, where $a_{j}$ is the length of the $j$ th edge of $H$. By the height of $H$ we mean $a_{d}$. By the width of $H$ we mean $a_{d-1}$. A hyperbox with $a_{d-1}>\lambda=1 / 3$ is called big, otherwise it is called small. Note that small hyperboxes satisfy $a_{d} \leq a_{d-1} \leq 1 / 3$. For each small hyperbox $H$, let $Q_{m, n}$ be an $r$-rectangle $\frac{1}{3 \cdot 2^{m}} \times \frac{1}{3 \cdot 2^{n}}$ such that $\frac{1}{3 \cdot 2^{m+1}}<a_{d-1} \leq \frac{1}{3 \cdot 2^{m}}$ and $\frac{1}{3 \cdot 2^{n+1}}<a_{d} \leq \frac{1}{3 \cdot 2^{n}}$. We say then that $H$ is of type $(m, n)$.

By an $(m, n)$-drawer we mean a hyperbox

$$
1 \times \cdots \times 1 \times \frac{1}{3 \cdot 2^{m}} \times \frac{1}{3 \cdot 2^{n}}
$$

Items of type $(m, n)$ will be packed into $(m, n)$-drawers. For example, item $H_{1}$ of type $(0,0)$ is packed into $(0,0)$-drawer, see Fig. 26 . The front wall of any $(m, n)$-drawer is the rectangle $\frac{1}{3 \cdot 2^{m}} \times \frac{1}{3 \cdot 2^{n}}$. We can also treat $B_{2}$ as the front wall of $[0,1]^{d}$. Drawers are created for packing items: there can be drawers of different sizes and many drawers of the same size, however at most one drawer of a fixed size is open at each stage of the packing process. Moreover, any two (open or closed) drawers have disjoint interiors.

For $d=1, D_{2}(1)$-algorithm is the Next Fit algorithm, i.e., we pack any item into the active bin as much to the left as it is possible; the average occupation in each bin is greater than $1 / 2$. For $d=2$, as $D_{2}(2)$-algorithm we use the method described in Zhang 
front wall

$H_{1}=0.6 \times 0.52 \times 0.28 \times 0.28 \times 0.21$

0.21

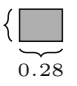

a drawer into which $H_{1}$ is packed

$1 / 3$

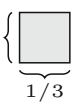

packing in a bin

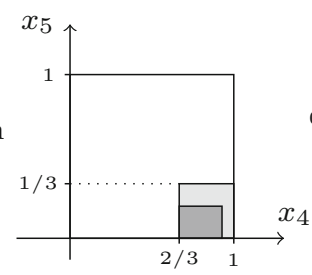

$(d-2)$-dimensional bottom
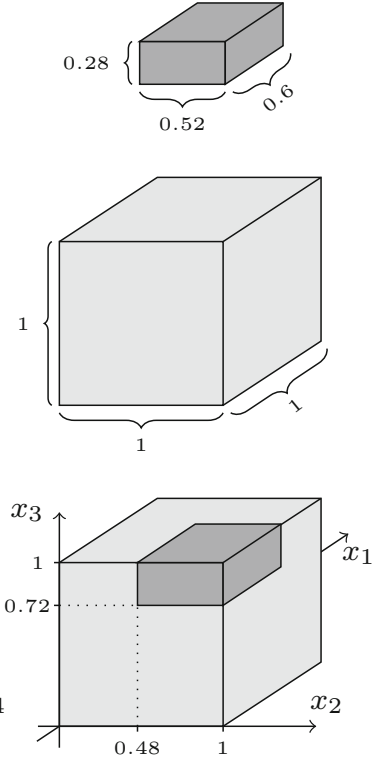

Fig. $26 d=5$ : a drawer $1 \times 1 \times 1 \times 1 / 3 \times 1 / 3$ is created in the active bin and an item $H_{1}=0.6 \times 0.52 \times$ $0.28 \times 0.28 \times 0.21$ is packed into it

et al. (2014); the average occupation in each bin is greater than 0.197. Similarly as for $D_{1}(d)$-algorithm, the reader can take any other 1-space bounded packing algorithm with the average occupation greater than $1 / 36$ for $d=1$ or greater than $1 / 108$ for $d=2$.

Assume that $d \geq 3$.

\section{$D_{2}(d)$-algorithm}

- If $H$ is big, then it is packed into the active bin from top to bottom along the right edge of the bin (see Sect. 4 for the definition) provided the interior of the packed item is disjoint with the union of open and closed drawers. If either $H$ should be packed so that its interior intersects a drawer that already contains a small hypercube or there is not enough empty space to pack $H$, then we close the active bin and open a new active bin to pack $\mathrm{H}$.

- If $H$ is a small hyperbox of type $(m, n)$, then

- it is packed into the open $(m, n)$-drawer in such a way that the $(d-2)$ dimensional bottom of $H$ is packed into the $(d-2)$-dimensional bottom of the drawer (i.e., into the $(d-2)$-dimensional unit cube) using the $D_{2}(d-2)$ algorithm.

- If there is not enough empty space in the open $(m, n)$-drawer to pack $H$, we close this drawer. A new drawer in opened in the following way. First, use the $\Lambda_{2}^{+}$-method to determine the proper rectangle to pack $Q_{m, n}$ into $B_{2}$, let it be called $Q$. The rectangle $Q$ is treated as an $r$-rectangle packed into $B_{2}$. 

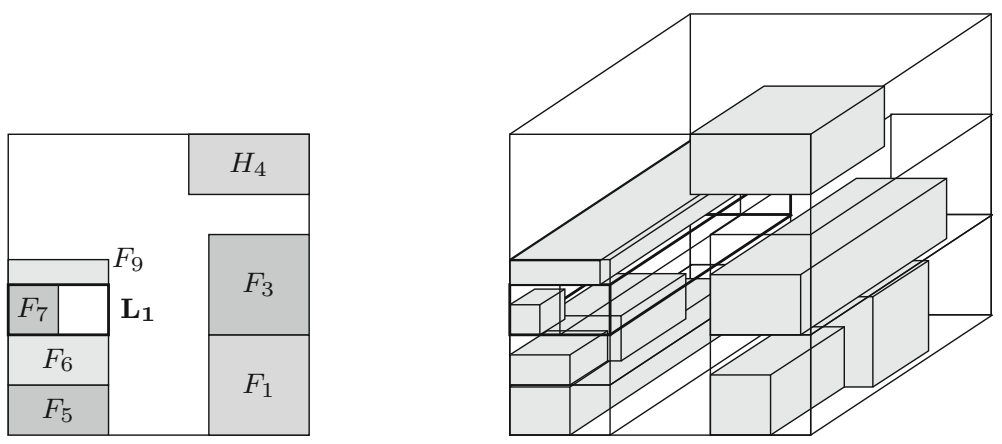

Fig. $27 D_{2}(d)$-algorithm

- If the drawer with the front wall equal to $Q$ is disjoint with the interior of any packed big item, then the new open $(m, n)$ - drawer is the one with the front wall equal to $Q$. The hyperbox $H$ is packed into this open drawer in such a way that the $(d-2)$-dimensional bottom of $H$ is packed into the $(d-2)$-dimensional bottom of the drawer using the $D_{2}(d-2)$-algorithm.

- If either there is no empty space in $B_{2}$ to pack $Q_{m, n}$ by $\Lambda_{2}^{+}$-method or the drawer with the front wall equal to $Q$ intersects the interior of a big packed item, then we close the active bin and open a new active bin to pack $\mathrm{H}$.

Example 12 Figure 27 illustrates $D_{2}(d)$-algorithm. The first three 3-dimensional boxes $H_{1}=0.4 \times 0.2 \times 0.2, H_{2}=0.3 \times 0.3 \times 0.3, H_{3}=0.8 \times 0.3 \times 0.2$ are of type $(0,0)$ and they are packed into $(0,0)$-drawers: items $H_{1}$ and $H_{2}$ into the drawer with the front wall $F_{1}$ while $H_{3}$ into the drawer with the front wall $F_{3}$ (there is not enough empty space to pack $H_{3}$ in the drawer with the front wall $F_{1}$ ). The next item is big; $H_{4}=0.4 \times 0.4 \times 0.2$ is packed at the top of the bin along its right edge. $H_{5}=0.9 \times 0.2 \times 0.16$ is of type $(0,1)$ and it is packed into the drawer with the front wall $F_{5} . H_{6}=0.2 \times 0.2 \times 0.1$ and $H_{8}=0.35 \times 0.25 \times 0.15$ are also of type $(0,1)$; they are packed into the drawer with the front wall $F_{6} . H_{7}=0.13 \times 0.1 \times 0.1$ is of type $(1,1)$ and it is packed into the drawer with the front wall $F_{7}$. This front wall is contained in the container $L_{1} . H_{9}=1 \times 0.3 \times 0.08$ is of type $(0,2)$ and it is packed into the drawer with the front wall $F_{9}$.

For $d \geq 3$ we will also use the notion of $b_{1}(i), b_{2}(i), b_{3}(i) . B_{2}$ is the front wall of the active bin, thus we can adopt the definitions of $b_{w}(i)$ for higher dimensions.

Theorem 2 The asymptotic competitive ratio for the $D_{2}(d)$-algorithm is not greater than $12 \cdot 3^{d}$.

Proof We show that the average occupation in each bin is greater than

$$
\vartheta_{d}=\frac{1}{12} \cdot\left(\frac{1}{3}\right)^{d}
$$


The proof is inductive. For $d=1$ the average occupation in each bin is greater than $1 / 2>(1 / 12) \cdot(1 / 3)$, for $d=2$, (see Zhang et al. 2014) it is greater than $0.197>(1 / 12) \cdot(1 / 3)^{2}$. Assume that $d \geq 3$ and that the statement holds in each dimension $n \in\{1,2, \ldots, d-1\}$.

Denote by $\vartheta(l)$ the total volume of items packed into the $l$ th bin $\mathcal{B}_{l}$. To prove that the average occupation in each bin is greater than $\vartheta_{d}$ it suffices to show that either $\vartheta(l)>\vartheta_{d}$ or $\vartheta(l)$ plus the volume of the first item that cannot be packed into $\mathcal{B}_{l}$ is greater than $2 \vartheta_{d}$.

Consider a few cases depending on the size of the first hyperbox item

$$
H_{u}=u_{1} \times \cdots \times u_{d-1} \times u_{d}
$$

that cannot be packed into $\mathcal{B}_{l}$.

Denote by $\omega$ the sum of volumes of open drawers. There is at most one open drawer of each size. The total volume of open drawers of width $1 / 3$ is smaller than $\frac{1}{3} \cdot \frac{1}{3}+\frac{1}{3} \cdot \frac{1}{6}+\frac{1}{3} \cdot \frac{1}{12}+\cdots=\frac{2}{9}$. The sum of volumes of open drawers of width $1 / 6$ is smaller than $\frac{1}{6} \cdot \frac{1}{6}+\frac{1}{6} \cdot \frac{1}{12}+\frac{1}{6} \cdot \frac{1}{24}+\cdots=\frac{2}{36}$. The total volume of open drawers of width $\lambda / 12$ is smaller than $\frac{1}{12} \cdot \frac{1}{12}+\frac{1}{12} \cdot \frac{1}{24}+\cdots=\frac{2}{144}$ and so on. Consequently,

$$
\omega<\frac{2}{9}+\frac{2}{36}+\frac{2}{144}+\cdots=\frac{2}{9} \cdot \frac{4}{3}=\frac{8}{27} \text {. }
$$

By the inductive assumption and by the fact that the smallest $r$-rectangle containing a rectangle $R=a_{d-1} \times a_{d}$ is of area not greater than 4 times the area of $R$, the average occupation in any closed $(m, n)$-drawer $L_{m, n}$ is greater than

$$
\frac{1}{4} \cdot \vartheta_{d-2} \cdot \operatorname{vol}\left(L_{m, n}\right)
$$

On Figs. 28, 29, 30 and 31 the front wall of an active bin is shown. We use grey or patterned rectangles to indicate one of the following things:

- front walls of big items;

- front walls of $(0, n)$-drawers;

- containers.

For example, on Fig. 28 the fifth and the sixth rectangle in $S_{1}$ (the left column) can be either front walls of $(0,1)$-drawers or containers as $L_{1}$ or $L_{2}$ on Fig. 25 . The patterned rectangle is the front wall of the big item that cannot be packed in the bin.

Denote by $h$ the sum of heights of big items packed into the active bin.

If $H_{u}$ is big and if $h+u_{d}>1 / 18$ (see Fig. 28), then

$$
\vartheta(l)+\operatorname{vol}\left(H_{u}\right)>\frac{1}{18} \cdot\left(\frac{1}{3}\right)^{d-1}=\frac{1}{6} \cdot\left(\frac{1}{3}\right)^{d}=2 \vartheta_{d} .
$$

In the next cases we will assume that $h+u_{d} \leq 1 / 18$ provided $H_{u}$ is big.

Case $1 \quad b_{\max }(u) \leq 17 / 18$ and $u_{d} \leq 1 / 6$. 


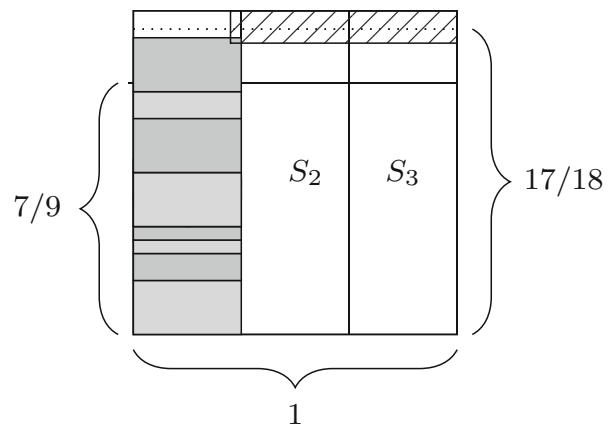

Fig. 28 Big items: $h+u_{d}>1 / 18$

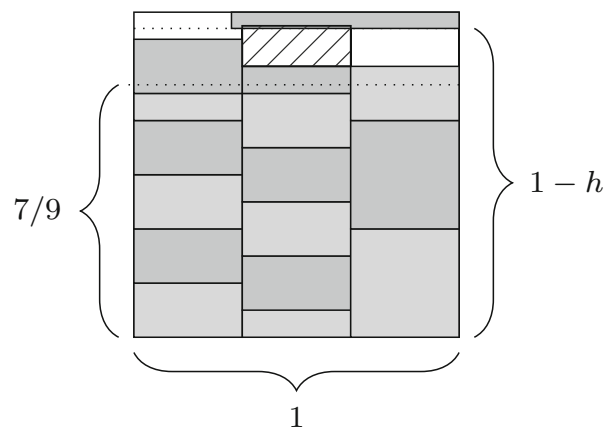

Fig. 29 Case 1

Since $b_{\max }(u) \leq 17 / 18$ (see Fig. 29), we can assume that $H_{u}$ is small; otherwise $h+u_{d}>1 / 18$.

The total volume of items packed into an open drawer can be close to 0 , thus we have to subtract the sum of volumes of open drawers $\omega<8 / 27$. Moreover, by Lemma 3, the area of empty space in $B_{2}$ is not greater than $\eta=\frac{2}{3} \cdot\left(\frac{1}{3}\right)^{2}$. Therefore the area of the part of $B_{2}$ covered by the front walls of open and closed drawers and, consequently, the volume of all open and closed drawers is greater than

$$
\frac{1}{3}\left[b_{1}(u)+b_{2}(u)+b_{3}(u)\right]-\eta .
$$

Since $b_{w}(u)>1-h-\frac{1}{6}$ for $w=1,2,3$, it follows that

$$
\begin{aligned}
\vartheta(l) & >\left(1-h-\frac{1}{6}-\omega-\eta\right) \cdot \frac{1}{4} \cdot \vartheta_{d-2}+h \cdot\left(\frac{1}{3}\right)^{d-1} \\
& >\left(1-\frac{1}{6}-\frac{8}{27}-\frac{2}{27}\right) \cdot \frac{1}{4} \cdot \frac{1}{12} \cdot\left(\frac{1}{3}\right)^{d-2}>\vartheta_{d} .
\end{aligned}
$$

Case $2 b_{\max }(u) \leq 17 / 18$ and $u_{d}>1 / 6$. Similarly as in Case 1 we can assume that $H_{u}$ is small. Since $u_{d-1} \geq u_{d}>1 / 6$, by the description of $\Lambda_{2}^{+}$method, $H_{u}$ should be packed into an open $(0,0)$-drawer (i.e., drawer whose front wall is a square 


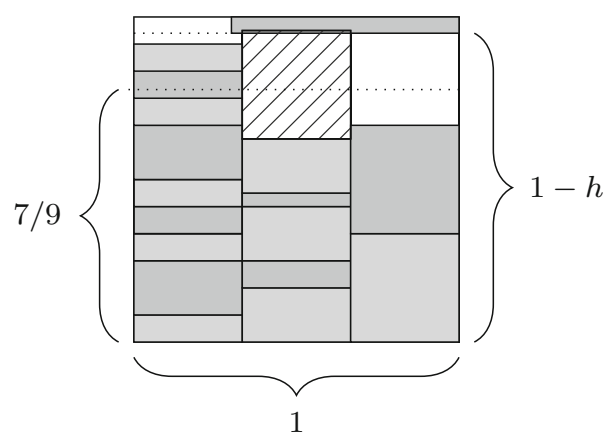

Fig. 30 Case 2

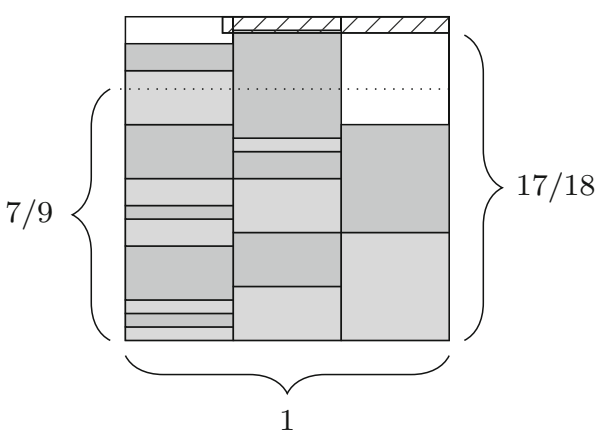

Fig. 31 Case 4

$1 / 3 \times 1 / 3)$. Since it is impossible, there is no open $(0,0)$-drawer. This implies that the total volume of open drawers is smaller than $\omega-\frac{1}{3} \cdot \frac{1}{3}$.

First assume that at least four $(0,0)$-drawers are closed. The total volume of small items is greater than $4 \cdot \frac{1}{9} \cdot \frac{1}{4} \cdot \vartheta_{d-2}=\vartheta_{d}$.

Now assume that at most three $(0,0)$-drawers are closed (see Fig. 30). By $b_{1}(u)>$ $\frac{17}{18}-\frac{1}{6}=\frac{7}{9}$ (at least one item of height not greater than $1 / 6$ was packed into $S_{2} \cup S_{3}$ ), by $b_{2}(u)>1-h-\frac{1}{3}$ as well as by $b_{3}(u)>1-h-\frac{1}{3}$ we deduce that

$$
\begin{aligned}
\vartheta(l) & >\left[\frac{7}{9} \cdot \frac{1}{3}+2\left(1-h-\frac{1}{3}\right) \cdot \frac{1}{3}-\left(\omega-\frac{1}{9}\right)-\eta\right] \cdot \frac{1}{4} \cdot \vartheta_{d-2}+h \cdot\left(\frac{1}{3}\right)^{d-1} \\
& >\left(\frac{7}{27}+2 \cdot \frac{2}{3} \cdot \frac{1}{3}-\frac{8}{27}+\frac{1}{9}-\frac{2}{27}\right) \cdot \frac{1}{4} \cdot \frac{1}{12} \cdot\left(\frac{1}{3}\right)^{d-2}=\vartheta_{d} .
\end{aligned}
$$

Case $3 b_{\max }(u)>17 / 18$ and $b_{\min }(u) \geq 7 / 9$. In this case

$$
\begin{aligned}
\vartheta(l) & >\left(2 \cdot \frac{7}{9} \cdot \frac{1}{3}+\frac{17}{18} \cdot \frac{1}{3}-\omega-\eta\right) \cdot \frac{1}{4} \cdot \vartheta_{d-2} \\
& >\left(2 \cdot \frac{7}{9} \cdot \frac{1}{3}+\frac{17}{18} \cdot \frac{1}{3}-\frac{8}{27}-\frac{2}{27}\right) \cdot \frac{9}{4} \cdot \frac{1}{12} \cdot\left(\frac{1}{3}\right)^{d}>\vartheta_{d}
\end{aligned}
$$


Case $4 b_{\max }(u)>17 / 18$ and $b_{\min }(u)<7 / 9$.

If at least four $(0,0)$-drawers are closed, then the total volume of small items is greater than $4 \cdot \frac{1}{9} \cdot \frac{1}{4} \cdot \vartheta_{d-2}=\vartheta_{d}$.

Now assume that at most three $(0,0)$-drawers are closed (see Fig. 31). Observe that at least one small item $R_{k}$ of height not greater than $1 / 6$ was packed into $S_{2}$ (otherwise $b_{\max }(u)<17 / 18$ ). Thus $7 / 9<b_{1}(k) \leq 17 / 18$. Moreover, all small items packed into $S_{3}$ have height greater than $1 / 6$. The reason is that if a small item $R_{l}$ of height not greater than $1 / 6$ was packed into $S_{3}$, then $7 / 9<b_{1}(l) \leq 17 / 18$ as well as $7 / 9<b_{2}(l) \leq 17 / 18$, which is a contradiction with $b_{\min }(u)<7 / 9$ and $b_{\max }(u)>17 / 18$. Consequently, at least two drawers of height $1 / 3$ were opened into $S_{3}$ and $b_{3}(u) \geq 2 / 3$. Moreover, $7 / 9 \leq b_{1}(u)<17 / 18$.

Subcase 4a: A drawer of height smaller than 1/6 (i.e., not greater that 1/12) was opened in $S_{2}$. This implies that $b_{1}(u)>\frac{17}{18}-\frac{1}{12}=\frac{31}{36}$ (otherwise this drawer should be created in $S_{1}$ ). Hence

$$
\begin{aligned}
\vartheta(l) & >\left(\frac{31}{36} \cdot \frac{1}{3}+\frac{17}{18} \cdot \frac{1}{3}+\frac{2}{3} \cdot \frac{1}{3}-\omega-\eta\right) \cdot \frac{1}{4} \cdot \vartheta_{d-2} \\
& >\left(\frac{31}{36} \cdot \frac{1}{3}+\frac{17}{18} \cdot \frac{1}{3}+\frac{2}{3} \cdot \frac{1}{3}-\frac{8}{27}-\frac{2}{27}\right) \cdot \frac{9}{4} \cdot \frac{1}{12} \cdot\left(\frac{1}{3}\right)^{d}>\vartheta_{d} .
\end{aligned}
$$

Subcase 4b: No drawer of height smaller than $1 / 6$ was opened in $S_{2}$. This implies that $b_{2}(u)$ is a multiple of $1 / 6$ (also $b_{3}(u)$ is a multiple of $1 / 6$ ).

If $b_{\max }(u)=b_{2}(u)$, then by $b_{\max }(u)>17 / 18$ we deduce that $b_{2}(u)=1$. As a consequence,

$$
\begin{aligned}
\vartheta(l) & >\left(\frac{7}{9} \cdot \frac{1}{3}+1 \cdot \frac{1}{3}+\frac{2}{3} \cdot \frac{1}{3}-\omega-\eta\right) \cdot \frac{1}{4} \cdot \vartheta_{d-2} \\
& >\left(\frac{7}{9} \cdot \frac{1}{3}+1 \cdot \frac{1}{3}+\frac{2}{3} \cdot \frac{1}{3}-\frac{8}{27}-\frac{2}{27}\right) \cdot \frac{9}{4} \cdot \frac{1}{12} \cdot\left(\frac{1}{3}\right)^{d}=\vartheta_{d} .
\end{aligned}
$$

If $b_{\max }(u)=b_{3}(u)$, then $b_{3}(u)=1$ and $b_{2}(u) \geq 2 / 3$. Hence

$$
\vartheta(l)>\left(\frac{7}{9} \cdot \frac{1}{3}+\frac{2}{3} \cdot \frac{1}{3}+1 \cdot \frac{1}{3}-\omega-\eta\right) \cdot \frac{1}{4} \cdot \vartheta_{d-2}=\vartheta_{d} .
$$

The average occupation in each bin is greater than $(1 / 12) \cdot(1 / 3)^{d}$. Consequently, the asymptotic competitive ratio of this packing strategy is not greater than $12 \cdot 3^{d}$.

Open Access This article is distributed under the terms of the Creative Commons Attribution 4.0 International License (http://creativecommons.org/licenses/by/4.0/), which permits unrestricted use, distribution, and reproduction in any medium, provided you give appropriate credit to the original author(s) and the source, provide a link to the Creative Commons license, and indicate if changes were made. 


\section{References}

Brown DJ (1979) A lower bound for on-line one-dimensional bin packing algorithms. Technical report R-864, Coordinated Sci. Lab, Urbana, Illinois

Coppersmith D, Raghavan P (1989) Multidimensional on-line bin packing: algorithms and worst-case analysis. Oper Res Lett 8(1):17-20. https://doi.org/10.1016/0167-6377(89)90027-8

Csirik J, Frenk J, Labb M (1993) Two-dimensional rectangle packing: on-line methods and results. Discrete Appl Math 45(3):197-204. https://doi.org/10.1016/0166-218X(93)90009-D

Epstein L, van Stee R (2005) Optimal online algorithms for multidimensional packing problems. SIAM J Comput 35(2):431-448. https://doi.org/10.1137/S0097539705446895

Grzegorek P, Januszewski J (2014) Online algorithms for 3-space bounded 2-dimensional bin packing and square packing. Rom J Inf Sci Technol 17(2):190-203

Grzegorek P, Januszewski J (2015) A note on one-space bounded square packing. Inf Process Lett 115(11):872-876. https://doi.org/10.1016/j.ipl.2015.06.001

Han X, Fujita S, Guo H (2001) A two-dimensional harmonic algorithm with performance ratio 2.7834. IPSJ SIG Notes 93:43-50

Han X, Chin FYL, Ting HF, Zhang G, Zhang Y (2011) A new upper bound 2.5545 on 2d online bin packing. ACM Trans Algorithms 7(4):50:1-50:18. https://doi.org/10.1145/2000807.2000818

Heydrich S, van Stee R (2016) Beating the harmonic lower bound for online bin packing. In: 43rd international colloquium on automata, languages, and programming, Schloss Dagstuhl-LZI, Wadern, LIPIcs Leibniz international proceedings in informatics, vol 55, pp 41:1-41:14

Januszewski J, Zielonka $Ł$ (2016) Improved online algorithms for 2-space bounded 2-dimensional bin packing. Int J Found Comput Sci 27(04):407-429. https://doi.org/10.1142/S0129054116500076

Januszewski J, Zielonka $Ł$ (2018) Online packing of rectangular items into square bins. In: Solis-Oba R, Fleischer R (eds) Approximation and online algorithms. Lecture notes in computer science. WAOA 2017, vol 10787. Springer, Cham, pp 147-163

Johnson DS (1974) Fast algorithms for bin packing. J Comput Syst Sci 8(3):272-314. https://doi.org/10. 1016/S0022-0000(74)80026-7

Johnson DS, Demers A, Ullman JD, Garey MR, Graham RL (1974) Worst-case performance bounds for simple one-dimensional packing algorithms. SIAM J Comput 3(4):299-325. https://doi.org/10.1137/ 0203025

Lee CC, Lee DT (1985) A simple on-line bin-packing algorithm. J ACM 32(3):562-572. https://doi.org/ $10.1145 / 3828.3833$

Liang FM (1980) A lower bound for on-line bin packing. Inf Process Lett 10(2):76-79. https://doi.org/10. 1016/S0020-0190(80)90077-0

Ramanan P, Brown DJ, Lee C, Lee D (1989) On-line bin packing in linear time. J Algorithms 10(3):305-326. https://doi.org/10.1016/0196-6774(89)90031-X

Seiden SS (2002) On the online bin packing problem. J ACM 49(5):640-671. https://doi.org/10.1145/ 585265.585269

Seiden SS, van Stee R (2003) New bounds for multidimensional packing. Algorithmica 36(3):261-293. https://doi.org/10.1007/s00453-003-1016-7

Ullman JD (1971) The performance of a memory allocation algorithm. Technical report 100, Princeton University, Department of Electrical Engineering, Computer Sciences Laboratory

van Vliet A (1992) An improved lower bound for on-line bin packing algorithms. Inf Process Lett 43(5):277284. https://doi.org/10.1016/0020-0190(92)90223-I

Woeginger G (1993) Improved space for bounded-space, on-line bin-packing. SIAM J Discrete Math 6(4):575-581. https://doi.org/10.1137/0406045

Yao ACC (1980) New algorithms for bin packing. J ACM 27(2):207-227. https://doi.org/10.1145/322186. 322187

Zhang Y, Chen J, Chin FYL, Han X, Ting HF, Tsin YH (2010a) Improved online algorithms for 1-space bounded 2-dimensional bin packing. Springer, Berlin, pp 242-253

Zhang Y, Chin FYL, Ting HF (2010b) One-space bounded algorithms for two-dimensional bin packing. Int J Found Comput Sci 21(06):875-891. https://doi.org/10.1142/S0129054110007611

Zhang Y, Chin FYL, Ting HF, Han X (2013) Online algorithms for 1-space bounded multi dimensional bin packing and hypercube packing. J Comb Optim 26(2):223-236. https://doi.org/10.1007/s10878-0129457-z 
Zhang Y, Chin FYL, Ting HF, Han X, Poon CK, Tsin YH, Ye D (2014) Online algorithms for 1-space bounded 2-dimensional bin packing and square packing. Theor Comput Sci 554:135-149. https://doi. org/10.1016/j.tcs.2014.02.045

Zhao X, Shen H (2015) On-line algorithms for 2-space bounded cube and hypercube packing. Tsinghua Sci Technol 20(3):255-263

Zielonka $Ł$ (2016) On-line packing cubes into n unit cubes. Bull Pol Acad Sci Math 64:185-198 